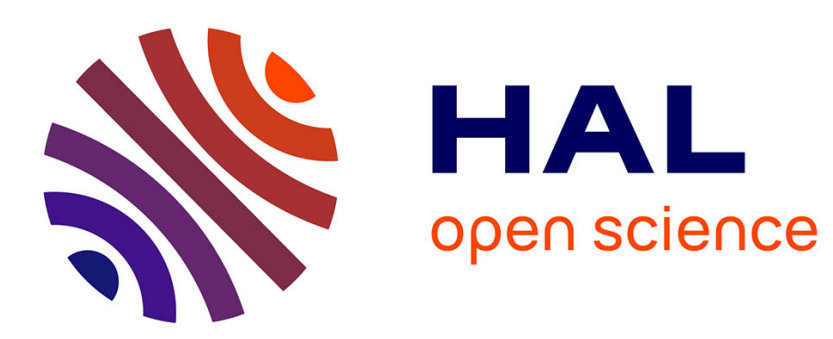

\title{
Motions, forces and mode transitions in vortex-induced vibrations at low mass-damping
}

Asif Khalak, Charles Williamson

\section{To cite this version:}

Asif Khalak, Charles Williamson. Motions, forces and mode transitions in vortex-induced vibrations at low mass-damping. Journal of Fluids and Structures, 1999, 13 (7-8), pp.813-851. 10.1006/jfls.1999.0236 . hal-03000247

\section{HAL Id: hal-03000247 \\ https://hal.science/hal-03000247}

Submitted on 12 Nov 2020

HAL is a multi-disciplinary open access archive for the deposit and dissemination of scientific research documents, whether they are published or not. The documents may come from teaching and research institutions in France or abroad, or from public or private research centers.
L'archive ouverte pluridisciplinaire HAL, est destinée au dépôt et à la diffusion de documents scientifiques de niveau recherche, publiés ou non, émanant des établissements d'enseignement et de recherche français ou étrangers, des laboratoires publics ou privés. 


\title{
MOTIONS, FORCES AND MODE TRANSITIONS IN VORTEX-INDUCED VIBRATIONS AT LOW MASS-DAMPING
}

\author{
A. KhalaK* and C. H. K. Williamson \\ Mechanical and Aerospace Engineering, Upson Hall, Cornell University, Ithaca, \\ NY 14853, U.S.A.
}

\begin{abstract}
These experiments, involving the transverse oscillations of an elastically mounted rigid cylinder at very low mass and damping, have shown that there exist two distinct types of response in such systems, depending on whether one has a low combined mass-damping parameter (low $m^{*} \zeta$ ), or a high mass-damping (high $m^{*} \zeta$ ). For our low $m^{*} \zeta$, we find three modes of response, which are denoted as an initial amplitude branch, an upper branch and a lower branch. For the classical Feng-type response, at high $m^{*} \zeta$, there exist only two response branches, namely the initial and lower branches. The peak amplitude of these vibrating systems is principally dependent on the mass-damping $\left(m^{*} \zeta\right)$, whereas the regime of synchronization (measured by the range of velocity $\left.U^{*}\right)$ is dependent primarily on the mass ratio, $m^{*}$. At low $\left(m^{*} \zeta\right)$, the transition between initial and upper response branches involves a hysteresis, which contrasts with the intermittent switching of modes found, using the Hilbert transform, for the transition between upper-lower branches. A $180^{\circ}$ jump in phase angle $\phi$ is found only when the flow jumps between the upper-lower branches of response. The good collapse of peak-amplitude data, over a wide range of mass ratios $\left(m^{*}=1-20\right)$, when plotted against $\left(m^{*}+C_{A}\right) \zeta$ in the "Griffin" plot, demonstrates that the use of a combined parameter is valid down to at least $\left(m^{*}+C_{A}\right) \zeta$ $\sim 0.006$. This is two orders of magnitude below the "limit" that had previously been stipulated in the literature, $\left(m^{*}+C_{A}\right) \zeta>0 \cdot 4$. Using the actual oscillating frequency $(f)$ rather than the still-water natural frequency $\left(f_{N}\right)$, to form a normalized velocity $\left(U^{*} / f^{*}\right)$, also called "true" reduced velocity in recent studies, we find an excellent collapse of data for a set of response amplitude plots, over a wide range of mass ratios $m^{*}$. Such a collapse of response plots cannot be predicted a priori, and appears to be the first time such a collapse of data sets has been made in free vibration. The response branches match very well the Williamson-Roshko (Williamson \& Roshko 1988) map of vortex wake patterns from forced vibration studies. Visualization of the modes indicates that the initial branch is associated with the $2 \mathrm{~S}$ mode of vortex formation, while the Lower branch corresponds with the $2 \mathrm{P}$ mode. Simultaneous measurements of lift and drag have been made with the displacement, and show a large amplification of maximum, mean and fluctuating forces on the body, which is not unexpected. It is possible to simply estimate the lift force and phase using the displacement amplitude and frequency. This approach is reasonable only for very low $m^{*}$. (C) 1999 Academic Press.
\end{abstract}

\section{REVIEW AND PRELIMINARY RESULTS}

VORTEX-INDUCED vibration of structures is of practical interest to many fields of engineering; for example, it can cause vibrations in heat exchanger tubes, it influences the dynamics of riser tubes bringing oil from the seabed to the surface, it is important to the design of civil

* Current address: Gas Turbine Laboratory, M.I.T., Cambridge, 02139, U.S.A. 
engineering structures such as bridges and chimneys stacks, as well as to the design of marine and land vehicles, and it can cause large-amplitude vibrations of tethered structures in the ocean. These examples are but a few in a large number of problems where vortexinduced vibrations (VIV) are important. The practical significance of vortex-induced vibration has led to a large number of fundamental studies, many of which are discussed in the comprehensive reviews of Sarpkaya (1979), Griffin \& Ramberg (1982), Bearman (1984), Parkinson (1989), and in the books by Blevins (1990), Naudascher \& Rockwell (1993), and Sumer \& Fredsøe (1997). In the present work, we are concerned with the transverse oscillations of an elastically mounted rigid cylinder, where there is presently a renewed interest to investigate experimental conditions of very low mass and damping, and to compare with the many direct numerical simulations (DNS) of this flow, now being conducted, where mass and damping can actually be set to zero. Related studies on flexible cylinders [for example, Vickery and Watkins (1964), Fujarra et al. (1998), Saltara et al. (1998), Pesce \& Fujarra (1999)], on articulated or pivoted cylinders [for example, Atsavapranee et al. (1998)], and on cylinders in a shear flow (Stansby 1976; Griffin 1985; Balasubramanian \& Skop 1996) have also received renewed attention, fuelled in part by the present interest in dynamics of offshore structures and tethered bodies.

In the relatively simple case of the elastically mounted cylinder there exist, however, some fundamental questions concerning vibration phenomena for very low mass and damping, for which there are very few laboratory investigations. Four of these questions, which shall form the main backbone to this introductory review and to the rest of this paper, and which are a stimulus to our ongoing research, are listed below.

(i) Combined mass-damping parameter: Under what conditions does the classically employed combined "mass-damping parameter" collapse peak amplitude data? What is the functional shape for a plot of peak amplitude versus mass-damping?

(ii) Maximum amplitude: What is the maximum amplitude attainable for a cylinder undergoing vortex-induced vibration, for conditions of extremely small mass and damping?

(iii) Response modes and mode jumps: What modes of response exist, and what are the corresponding wake vortex dynamics? How (and why) do the flow transitions occur between different modes?

(iv) Definition of synchronization: How does one usefully define synchronization or lock-in for this problem?

Before addressing these questions in the light of previous investigations and the present work, we need first to introduce an equation of motion generally used to represent the vortex-induced vibrations of a cylinder oscillating in the transverse $y$-direction (normal to the flow), as follows:

$$
m \ddot{y}+c \dot{y}+k y=F,
$$

where $m$ is the total oscillating structural mass, $c$ the structural damping, $k$ the spring constant, and $F$ the fluid force in the transverse direction. In the regime where the body oscillation frequency is synchronized with the periodic vortex wake mode (or synchronized with the frequency of induced force on the body), a good approximation to the force and the response is given by

$$
\begin{aligned}
& F(t)=F_{0} \sin (\omega t+\phi), \\
& y(t)=y_{0} \sin (\omega t),
\end{aligned}
$$

where $\omega=2 \pi f$, and $f$ is the body oscillation frequency. We shall see shortly that the phase angle $\phi$ is vitally important in governing the body response in this problem, as has been shown clearly in the many reviews [e.g. Sarpkaya (1979), Bearman (1984)]. The response of 
TABLE 1

Nondimensional groups

$\begin{array}{lcc}\text { Mass ratio } & m^{*} & \frac{m}{\pi \rho D^{2} L / 4} \\ \text { Damping ratio } & \zeta & \frac{c}{2 \sqrt{k\left(m+m_{A}\right)}} \\ \text { Velocity ratio } & U^{*} & \frac{U}{f_{N} D} \\ \text { Amplitude ratio } & A^{*} & \frac{y_{o}}{D} \\ \text { Frequency ratio } & f^{*} & \frac{f}{f_{N}} \\ \text { Streamwise force coefficient } & C_{X} & \frac{F_{X}}{\frac{1}{2} \rho U^{2} \mathrm{DL}} \\ \text { Transverse force coefficient } & C_{Y} & \frac{F_{Y}}{\frac{1}{2} \rho U^{2} D L} \\ \text { Reynolds number } & \mathrm{Re} & \frac{\rho U D}{v}\end{array}$

Notes regarding these groups

(i) We choose here to use $f_{N}$ as the natural frequency in water, and correspondingly use $\zeta$ as the ratio of (structural damping)/(critical damping in water). The frequency $f$, used in $f^{*}$, is the actual body oscillating frequency during induced vibration.

(ii) The added mass is $m_{A}=C_{A} m_{d}$, where $m_{d}$ is the displaced mass of fluid $=\pi \rho D^{2} L / 4$, and where $L$ is the cylinder length. $C_{A}$ is the added mass coefficient for small transverse oscillations in otherwise still fluid, and experiments yield values $C_{A} \approx 1 \cdot 0$ to within $5 \%$, supporting the use of the inviscid flow coefficient $C_{\mathrm{A}}=1$. (This $C_{\mathrm{A}}$ should not be confused with the transverse force coefficient often measured in phase with the acceleration during flow-induced vibration, which of course is a function of amplitude and frequency of vibration, etc.)

(iii) The coordinate system $(x, y, z)$ has its origin on the body axis at the lowest position of the cylinder: $x$ is measured downstream, $y$ is measured normal to the flow, and $z$ is measured upwards along the vertical cylinder axis.

the cylinder in the transverse direction is influenced by the set of nondimensional groups defined in Table 1.

The response amplitude and frequency may be derived in a straightforward manner from equations (1)-(3), as done by several previous investigators (see the review papers for example). Here we shall simply formulate the equations, in a somewhat different manner, in terms of our chosen nondimensional parameters (see Table 1) as follows:

$$
\begin{gathered}
A^{*}=\frac{1}{4 \pi^{3}} \frac{C_{Y} \sin \phi}{\left(m^{*}+C_{A}\right) \zeta}\left(\frac{U^{*}}{f^{*}}\right)^{2} f^{*}, \\
f^{*}=\sqrt{\frac{m^{*}+C_{A}}{m^{*}+C_{E A}}},
\end{gathered}
$$


where $C_{A}$ is the potential added mass coefficient, and $C_{E A}$ is an "effective" added mass coefficent that includes an apparent effect due to the total transverse fluid force in-phase with the body acceleration $\left(C_{Y} \cos \phi\right)$ :

$$
C_{E A}=\frac{1}{2 \pi^{3}} \frac{C_{Y} \cos \phi}{A^{*}}\left(\frac{U^{*}}{f^{*}}\right)^{2} .
$$

It should be noted that the amplitude $A^{*}$ is proportional to the transverse force component that is in phase with the body velocity $\left(C_{Y} \sin \phi\right)$, and we shall see experimentally that, for small mass and damping, the precise value of the phase angle $\phi$ has a large effect on the response amplitude.

One of the most fundamental questions listed at the start of the introductory review is the first: How is the maximum response amplitude a function of system mass and damping? Generally, in the literature, this information has been plotted as $A_{\max }^{*}$ versus a parameter, $S_{G}$, proportional to the product of mass and damping, following the first comprehensive compilation of existing data by Griffin and co-workers in the 1970s, and labelled here for convenience as the "Griffin" plot. This classical plot has recently been updated by Skop \& Balasubramanian (1997), and their compiled data is included in Figure 1, showing the characteristic shape whereby the amplitude reaches some limiting value as mass and damping (or $S_{G}$ ) become small. The logic in choosing a combined parameter involving the product of mass and damping comes from observation of equation (4) for $A^{*}$, and has been discussed in many previous papers. Bearman (1984), for example, discusses in his review that for large mass ratios $\left(m^{*} \gg 1\right)$, the actual cylinder oscillation frequency $(f)$ at resonance will be close to the vortex-shedding frequency for the static cylinder $\left(f_{0}\right)$, and also close to the system natural frequency $\left(f_{N}\right)$, i.e. $f \approx f_{O} \approx f_{N}$, and thus $f^{*} \approx 1 \cdot 0$ [see equation (5), for large $\left.m^{*}\right]$. Thus at resonance, the parameter $\left(U^{*} / f^{*}\right)=(U / f D) \approx\left(U / f_{O} D\right)=1 / S$, where $S$ is the Strouhal number of the static cylinder, yielding an expected resonance of cylinder response, over a wide range of $\mathrm{Re}$, to occur at the normalized velocity, $U^{*} \approx 5-6$. The assumption is therefore often made that both $\left(U^{*} / f^{*}\right)$ and $f^{*}$ are constants, under resonance conditions, giving

$$
A_{\max }^{*} \propto \frac{C_{Y} \sin \phi}{\left(m^{*}+C_{A}\right) \zeta} .
$$

One should note that, if $\left(U^{*} / f^{*}\right)$ is assumed constant, the excitation $\left(C_{Y} \sin \phi\right)$ is a function of $\left\{A^{*}\right\}$ only. Therefore, under these assumptions, $A_{\max }^{*}$ is a function only of the product of mass and damping $\left(m^{*}+C_{A}\right) \zeta$. Incidentally, one may then derive, from the Griffin plot data in Figure 1, the functional relationship between $\left(C_{Y} \sin \phi\right)$ and $A_{\max }^{*}$. One must stress that equation (7) is of course dependent on the earlier assumptions remaining reasonable (i.e. $f^{*} \approx 1 \cdot 0$ ), which is not self-evident over a large range of mass and damping.

A number of mass-damping parameters have been introduced and are in general use. Vickery \& Watkins (1964), who considered an equation of motion for flexible cantilevers, plotted their peak amplitudes versus a "stability parameter", $K_{S}$, defined as

$$
\text { stability parameter }=K_{S}=\pi^{2}\left(m^{*} \zeta\right) \text {. }
$$

Scruton (1965) used a parameter, proportional to $K_{S}$, for his experiments on elastically mounted cylinders, which has since been termed the Scruton number, $[\mathrm{Sc}]$ :

$$
\text { Scruton number }=\mathrm{Sc}=\frac{\pi}{2}\left(m^{*}\right)
$$


A slightly different parameter was independently derived from a response analysis involving the van der Pol equation by Skop and Griffin (1973), and they began to compile data from several different experiments as a means to usefully predict response amplitudes. The combined response parameter was subsequently termed $S_{G}$ in Skop (1974), and we define it here as follows:

$$
\text { Skop-Griffin parameter }=S_{G}=2 \pi^{3} S^{2}\left(m^{*}\right) \text {. }
$$

The first extensive compilations of many different investigations was made in Griffin et al. (1975), using $S_{G}$, and subsequently the classical log-log form of the plot (Griffin 1980) has become the widely used presentation of peak response data. An updated version of the "Griffin" plot by Skop \& Balasubramanian (1997) is shown in Figure 1, although here we have chosen to replot the data using a linear vertical axis.

Despite the extensive use of the log-log "Griffin" plot by practicing engineers (it is included for example in the standard codes of Det Norske Veritas), it is not known precisely under what conditions the assumptions regarding $\left(U^{*} / f^{*}\right)$ and $f^{*}$ would hold, that would lead to a "unique" curve of $A_{\max }^{*}$ versus $S_{G}$. Problems regarding the validity of this widely used plot were pointed out clearly by Sarpkaya $(1978,1979)$ and by Bearman (1984), and others. Bearman states that, in the case of small $m^{*}$, even if $m^{*} \zeta$, or equivalently $\left(m^{*}+C_{A}\right) \zeta$, is kept constant, the frequency $f^{*}$ will be affected by independent variations of mass ratio $m^{*}$, as evident from our equation (5). Therefore, one might expect that the peak response $A_{\max }^{*}$ will not be a unique function of the combined $m^{*} \zeta$ parameter. However, the effects of individual variation of $m^{*}$, for example, might be negligibly small, under some circumstances. This raises the important question, which we shall address in the present paper: What range of values of $m^{*}$ and $\zeta$ will yield, to good accuracy, a single relation between $A_{\max }^{*}$ and $m^{*} \zeta$ ?

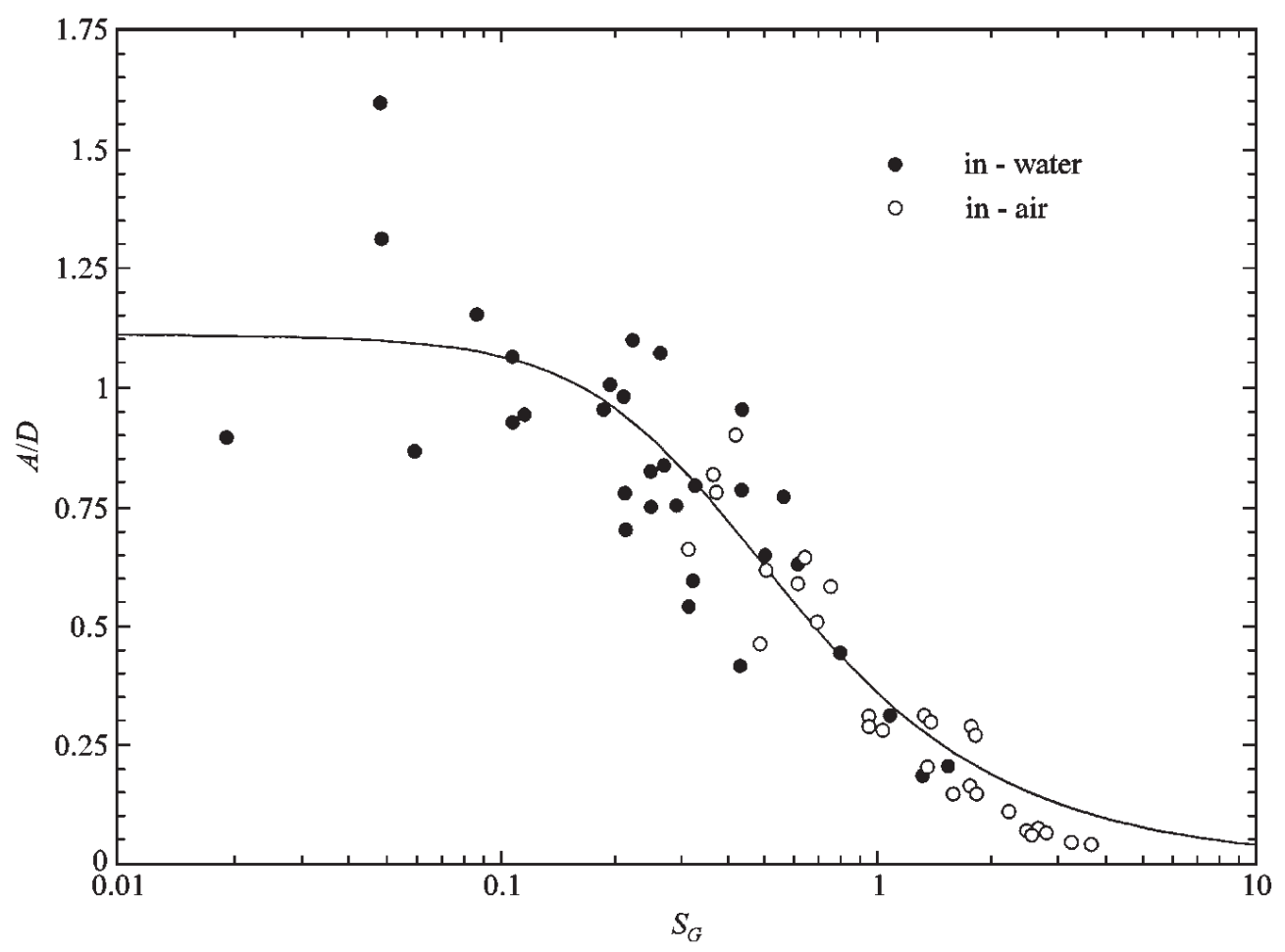

Figure 1. "Griffin" plot showing peak ampltude $A^{*}$ versus the Skop-Griffin parameter $S_{G}$. This plot differs from those that have typically been used, since they were introduced by Griffin et al. (1975), in that we use a linear axis for the ampltude $A^{*}$. This scaling reveals a large scatter in the amplitude, which was otherwise not evident in the classical log-log plots. The curve fit comes from a recent update by Skop \& Balsubramanian (1997). 
Sarpkaya $(1978,1979)$ originally brought this significant question sharply into focus, and stated that simple observation of his equation of motion [equivalent to equation (1)] showed immediately that the response of the system is independently governed by mass and damping. By analysing three pairs of low-amplitude response data (Sarpkaya 1978), with each pair at similar $S_{G}$ but different $m^{*}$ values, he calculated that there is a large (50-100\%) influence of mass ratio on $A_{\text {max }}^{*}$, even for large $S_{G} \approx 2 \cdot 0$. He then stated that one should use the combined parameter $S_{G}$ only if $S_{G}>1 \cdot 0$. In later work, Sarpkaya $(1993,1995)$ further states that it is routinely assumed that $S_{G}$ (involving the product $m^{*} \zeta$ ) is a universal parameter to embody the effects of both mass and damping, even though the inadvisability of such a procedure has already been seriously questioned. He states: One must conclude that the dynamic response is governed, among other parameters, by $m^{*}$ and $\zeta$ independently, not just by $\left(m^{*} \zeta\right)$. This point is supported by Zdravkovich (1990), who states that the Scruton number, involving $m^{*} \zeta$, is useful for wind engineering $\left(m^{*}>100\right)$, but that the use of the Scruton number is not legitimate for marine and offshore engineering $\left(m^{*}<10\right)$. He states that the mass ratio and damping should be treated as separate parameters in marine and offshore engineering applications.

On the other hand, Ramberg \& Griffin (1981) report two sets of experimental data, each for the same value of $S_{G}=0 \cdot 5-0 \cdot 6$, but where one experiment is conducted in air and the other in water, ensuring a factor of 10 between the mass ratios; $m^{*}=3.8$ and 34.0 respectively. These data demonstrate two points. Firstly, the lower mass ratio leads to a wider synchronization regime, extending over a larger range of normalized velocity $U^{*}$ (note also our results in Figure 2). Secondly, at the same $S_{G}$, the peak amplitude is roughly unchanged at $A_{\max }^{*}=0 \cdot 5$. Thus, even at reasonably low mass ratios of order (10), the peak amplitude is found to collapse well with the combined mass-damping, and this occurs at a value of $S_{G}$ below Sarpkaya's accepted range of $S_{G}>1 \cdot 0$. Naudascher \& Rockwell (1993) also conclude that the compilation of Griffin and co-workers shows that, with a certain scatter, the unique relationship between $A^{*}$ and $S_{G}$ extends even to very small values of $S_{G}$. However, there is quite a large scatter in the data, as is apparent in Figure 1, and based on existing plots it is presently difficult to support the proposal for a unique relationship to very low $S_{G}$.

It seems evident that the question regarding the range of mass and damping over which a combined parameter will predict well the peak amplitude has not been answered to date. Sarpkaya's $(1978,1979)$ criticism over the use of the combined parameter has a good basis, as is clear by inspection of the equation for $A_{\max }^{*}$ given in equation (4) and the earlier discussion, although it is also apparent that his stipulation that a combined parameter is only useful above $S_{G}>1.0$ is possibly too stringent. In the Griffin plot, there are almost no data points corresponding to $S_{G}>1 \cdot 0$, so that this condition would appear to rule out almost the complete plot in Figure 1. This open question is addressed in the present work, and indeed in our ongoing research, and we shall see that the single parameter $\left(m^{*} \zeta\right)$ is good at collapsing peak response data, over a very wide range of $\left(m^{*} \zeta\right)$ and $m^{*}$.

Equations to fit the compiled data in the Griffin plot have been put forward by a number of investigators [whose rather complicated equations are listed in Table 3-2 of Blevins (1990)]. Sarpkaya (1978) used the assumed equation of motion to more rigorously derive a simple equation relating $A_{\max }^{*}$ to $S_{G}$, as follows:

$$
A_{\max }^{*}=\frac{B}{\sqrt{C+S_{G}^{2}}} .
$$

This equation fits the existing data reasonably well, as indicated by the curve in Figure 1 (where $B=0.385$ and $C=0 \cdot 12$ ), from the recent update of compiled data by Skop $\&$ 
Balsubramanian (1997). It should be noted, however, that $B$ and $C$ are not constants. The value of $B$ is proportional to force coefficient $C_{Y}$, for example, and Sarpkaya noted that $C_{Y}$ evidently depends on $\mathrm{A}^{*}$ (in fact, it strongly depends on $A^{*}$ ). Therefore, one cannot assume that such a formulation will accurately fit the data, a priori. In fact, an even simpler formulation is put forward in Triantafyllou \& Grosenbaugh (1995), where they empirically represent $C_{y} \sin \phi$ as a linear function of $A^{*}$ :

$$
A_{\mathrm{max}}^{*}=\frac{D}{E+S_{G}},
$$

which also fits the Griffin plot data reasonably well. The question of how well such formulations fit the data, when $A^{*}$ is evaluated systematically over a range of mass and damping in a single set of experiments, has not been addressed before, and this provides part of the impetus for the present and ongoing work.

We now return to the second question addressed at the start of Section 1, which is perhaps the most basic question one may ask of such an elastically mounted system: What is the absolute largest attainable amplitude? Despite the very large number of investigations on this problem, the precise answer to this question does not appear to be known. An estimate of this largest amplitude is given by the limit of $A^{*}$ as mass and damping tend to zero, or in the case of DNS computations, when $m^{*}$ or $\zeta$ are set precisely to zero.

Peak amplitudes, evaluated from the curve fits in the "Griffin" plots under the limit of zero mass-damping, yield values of $A^{*}=1 \cdot 3$ (Griffin \& Ramberg 1982; Sarpkaya 1978), whereas the most recent update of compiled data by Skop \& Balasubramanian (1997) gives $A^{*}=1 \cdot 1$, as indicated in Figure 1. From both forced oscillations and "equivalent" free vibration experiments, Hover et al. $(1997,1998)$ give maximum possible amplitudes for zero mass-damping as $A^{*}=0 \cdot 8$. Predictions of the peak amplitude are also given by plots of maximum "excitation" $C_{y} \sin \phi$ versus $A^{*}$ [see Griffin (1980), Bearman's (1984) Figure 5]. The peak expected amplitude for zero damping is to be found when the excitation $C_{y} \sin \phi$ is also zero, which is found when roughly $A^{*}=1 \cdot 5$, as mentioned by Bearman (1984) and by Naudascher \& Rockwell (1993). From the above evidence, one may conclude that the largest attainable amplitude lies anywhere between 0.8 and 1.5 . The large disparity concerning this amplitude limit was the subject of much debate at the recent Conference on Bluff Body Wakes and Vortex-Induced Vibration (Washington, DC, June 1998); see Bearman \& Williamson (1998).

By studying the data listed here, we conclude that the surprisingly large range of typically quoted peak amplitude is caused by the inherent large scatter in the existing data, and also by the fact that the Griffin plot and the excitation plot (involving $C_{y} \sin \phi$ ), both traditionally used for prediction purposes, actually comprise data for cantilevers and for pivoted cylinders, as well as for elastically mounted cylinders. Compiling all of these results, from quite different physical experiments, into a single plot, with the hope that a single curve of data may ensue, might not therefore be reasonable.

To clarify this point, we present, in Table 2, an updated compilation of peak amplitude data from previous experiments conducted in water facilities (only), where mass ratios $m^{*}$ are typically much smaller (and responses typically larger) than found in air facilities. Much of the data is very recent, and we also include direct numerical simulations (DNS) results. Some deductions may be made from this assembled data; for example, Table 2(A) suggests that in no experiment for elastically mounted rigid cylinders to-date, does the peak amplitude actually exceed $A^{*}=1 \cdot 13$. Correspondingly, the data in Table $2(\mathrm{C})$, showing the peak amplitudes at which the excitation force $\left(C_{y} \sin \phi\right)$ for oscillating rigid cylinders becomes zero, also indicates an upper limit, for all the previous studies, of roughly $A^{*}=1 \cdot 1$. 
TABLE 2

Peak amplitude data

\begin{tabular}{|c|c|c|c|c|c|}
\hline Investigators & Year & Medium & Reynolds number & $m * \zeta$ & $\operatorname{Peak}(\mathrm{A} / \mathrm{D})$ \\
\hline \multicolumn{6}{|c|}{ (A) Elastically mounted rigid cylinders } \\
\hline Angrilli et al. & 1974 & Water & $2500-7000$ & $0 \cdot 049$ & $0 \cdot 54$ \\
\hline Dean et al. ${ }^{1}$ & 1977 & Water & $2800-10200$ & $0 \cdot 0055$ & $0 \cdot 94$ \\
\hline Moe \& Overvik & 1982 & Water & $6000-30000$ & $0 \cdot 013$ & $1 \cdot 09$ \\
\hline Anand \& Torum ${ }^{2}$ & 1985 & Water & $6500-35000$ & $0 \cdot 0132$ & $1 \cdot 07$ \\
\hline Sarpkaya & 1995 & Water & $6000-35000$ & $0 \cdot 052$ & $0 \cdot 95$ \\
\hline Fujarra et al. & 1998 & Water & $14410-50380$ & $0 \cdot 036$ & $1 \cdot 01$ \\
\hline Gharib et al. & 1998 & Water & $11000-40000$ & $0 \cdot 094$ & $0 \cdot 84$ \\
\hline Vikestad & 1998 & Water & $50000-100000$ & $0 \cdot 012$ & $1 \cdot 13$ \\
\hline Hover et al. $^{3}$ & 1998 & Water & 3800 & $0 \cdot 04$ & $0 \cdot 80$ \\
\hline \multicolumn{6}{|l|}{ (B) Cantilevers } \\
\hline Vickery \& Watkins ${ }^{4,5}$ & 1964 & Water & 7000 & $0 \cdot 0162$ & $1 \cdot 46$ \\
\hline King $^{5}$ & 1974 & Water & $6000-22500$ & $0 \cdot 032$ & $1 \cdot 60$ \\
\hline Pesce \& Fujarra ${ }^{5}$ & 1999 & Water & $6000-40000$ & $0 \cdot 016$ & $1 \cdot 32$ \\
\hline \multicolumn{6}{|c|}{ (C) Forced oscillations (Amplitude limit of positive excitation) } \\
\hline Mercier & 1973 & Water & $2000-33000$ & & $1 \cdot 10$ \\
\hline Sarpkaya & 1978 & Water & $5000-25000$ & & $0 \cdot 90$ \\
\hline Hover et al. ${ }^{3}$ & 1998 & Water & 3800 & & $0 \cdot 82$ \\
\hline \multicolumn{6}{|l|}{ (D) Low-Re experiments } \\
\hline \multicolumn{6}{|l|}{ Anagnostopoulos \& } \\
\hline Bearman & 1992 & Water & $90-150$ & $0 \cdot 179$ & $0 \cdot 55$ \\
\hline \multicolumn{6}{|c|}{ (E) Direct numerical simulation (DNS) } \\
\hline \multicolumn{6}{|c|}{ Blackburn \& } \\
\hline Karniadakis ${ }^{6}$ & 1993 & 2-D code & 200 & $0 \cdot 012$ & $0 \cdot 64$ \\
\hline \multicolumn{6}{|l|}{ Newman \& } \\
\hline Karniadakis ${ }^{6}$ & 1996 & 2-D code & 100 & $0 \cdot 00$ & $0 \cdot 65$ \\
\hline Shiels et al. & 1998 & 2-D code & 100 & $0 \cdot 00$ & $0 \cdot 59$ \\
\hline Fujarra et al. & 1998 & 2-D code & 200 & $\sim 0.015$ & $0 \cdot 61$ \\
\hline \multicolumn{6}{|l|}{ Evangelinos \& } \\
\hline Karniadakis & 1998 & $3-\mathrm{D}$ code & 1000 & $0 \cdot 00$ & $0 \cdot 74$ \\
\hline \multicolumn{6}{|c|}{ (F) Large-Eddy-Simulation (LES) } \\
\hline Saltara et al. & 1998 & 2-D code & 1000 & $0 \cdot 013$ & 0.67 \\
\hline
\end{tabular}

Notes regarding these collected data

${ }^{1}$ Amplitude response plots show multiple peaks, and large scatter.

${ }^{2}$ Cylinder oscillates vertically above a horizontal boundary, which is at three diameters below equilibrium position.

3 "Virtual" free vibration experiments using force feedback.

${ }^{4}$ Vickery and Watkins performed experiments with cantilevers, and not pivoted cylinders (as usually quoted).

${ }^{5}$ The peak A/D here is less than the tip amplitude, and involves a scaling using a modal parameter as defined in Griffin, et al. (1975).

${ }^{6} X-Y$ motions were simulated (two degrees of freedom).

This peak value is clearly much lower than the values often quoted as $A^{*}=1 \cdot 5$. One may conclude that the latter overestimation must be influenced by the data for cantilevers, pivoted rods, etc., for which the peak amplitudes are significantly higher $\left[A^{*} \approx 1 \cdot 6\right.$; see Table 2(B)]. A major part of the impetus for the present paper, and for our ongoing work, 
has been triggered by the need to precisely determine the maximum possible amplitude at very small mass and damping.

It should also be noted from the data in Table 2(E,F) that DNS computations of vortex-induced vibrations all yield a maximum of around $A^{*}=0 \cdot 6-0 \cdot 7$, which is significantly lower than the peak response found in experiment. One possibility is that this flow is Reynolds number dependent for the low Reynolds numbers, $\mathrm{Re}=100-200$, typically used in the simulations. (This is contrasted with typical values of $\mathrm{Re}=10^{3}-10^{5}$ for the experimental data). Support for this present suggestion of a Reynolds number effect comes also from the experiments of Anagnostopoulos \& Bearman (1992), who find low peak response similar to the simulations, $A^{*}=0 \cdot 55$, when they use similar low Reynolds numbers in the range, $\mathrm{Re}=90-150$. This disparity between high-Re experiment and low-Re simulation will be further discussed in the present paper, in the light of our own response measurements, and remains a very interesting unanswered question.

We shall now consider the third question posed at the start of Section 1, namely the question: What modes of response exist, as a function of mass ratio and damping? Previous investigations of the response amplitude as a function of normalized velocity $U^{*}$ revealed, not only a resonance near the condition where $f \approx f_{\mathrm{O}} \approx f_{N}$, but also a hysteresis whereby the amplitude could jump between a lower and upper value over a small range of $U^{*}$. This was demonstrated clearly by Feng (1968), whose smallest-damped response plot is shown in Figure 2. Subsequently, such a hysteresis was found also for a vibrating cable by Brika \& Laneville (1993), and for a flexible cantilever by Saltara et al. (1998) and Pesce \& Fujarra (1999). The mass ratio in Feng's experiments was of the order of 250.

For our much lower mass ratios, in this case $m^{*}=10 \cdot 1$, and for a value of $m^{*} \zeta$ here of around $4 \%$ of Feng's value, the response plot is distinctly different, as shown also in Figure 2.

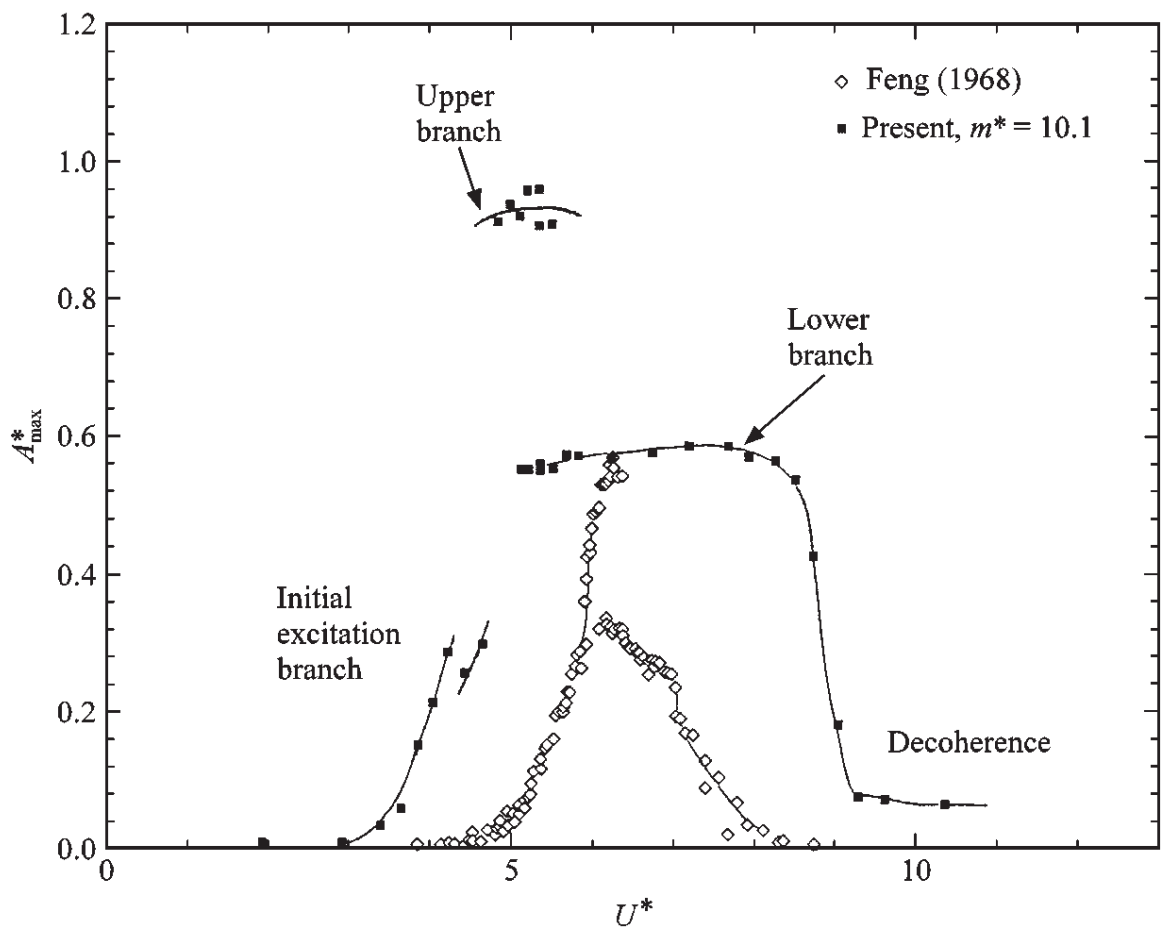

Figure 2. Amplitude response plot $A_{\max }^{*}$ versus normalized velocity $U^{*}$, for $m^{*} \zeta=0 \cdot 013$. This shows clearly the three response branches (initial, upper and lower). This low- $\left(m^{*} \zeta\right)$ type of response (for $m^{*}=10 \cdot 1$ ) is distinct from the type of high- $\left(m^{*} \zeta\right)$ plot (for $\left.m^{*}=248\right)$ of Feng (1968), where there are only two branches (initial and lower). We also note the much larger peak amplitude and regime of synchronization (measured along the $U^{*}$-axis). 

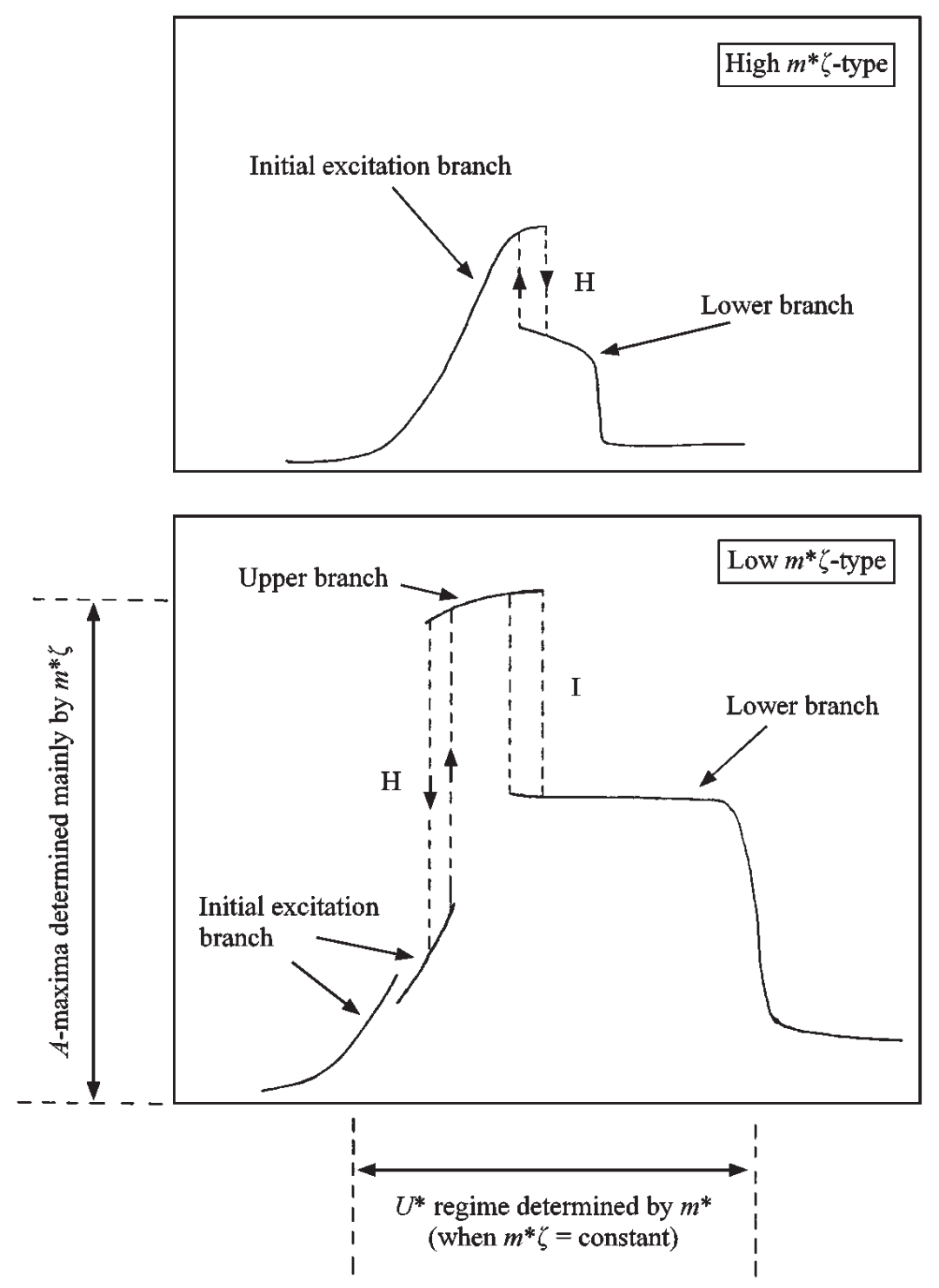

Figure 3. The two distinct types of amplitude response are shown here schematically. (Vertical axes represent $A^{*}$ and horizontal axes represent $U^{*}$.) The Feng-type of high- $\left(m^{*} \zeta\right)$ response exhibits only two branches (initial and lower), while our low- $\left(m^{*} \zeta\right)$ type of response exhibits three branches (initial, upper and lower). The mode transitions (see section 5) are either hysteretic $(\mathrm{H})$ or intermittently switching modes (I). The range of $U^{*}$ for synchronisation is controlled primarily by $m^{*}$ (when $m^{*} \zeta$ is constant), whereas the peak amplitudes are controlled principally by the product $m^{*} \zeta$.

The magnitude of the amplitude response is clearly much higher than in Feng's experiments in air, and the extent of $U^{*}$ over which there is significant response is 4 times larger than found by Feng, both of which are trends that might be expected. However, the character of the response for low mass-damping is also quite different, as we show schematically in Figure 3. For high- $\left(m^{*} \zeta\right)$ experiments, as in Feng, there are two solution branches only, which we shall define here, as follows: the "initial branch", which yields the highest amplitudes, and the "lower branch", with apparently a hysteresis between the two modes. Somewhat different mode transitions were shown in Brika \& Laneville (1993) in their vibrating cable experiments, but they also provide evidence for the existence of only an initial and lower branch, for high- $\left(m^{*} \zeta\right)$. On the other hand, the low- $\left(m^{*} \zeta\right)$ type of response in this work is characterized by not only the initial branch and the lower branch, but also by a separate upper branch. The existence of the three distinct branches was shown in Khalak \& Williamson (1996, 1997a, b). 
It is most interesting and relevant to mention here some early experiments of Meier-Windhorst (1939), who measured the response of a very short cylinder section partially immersed in a shallow "water table" flow, giving a length/diameter of only $1 \cdot 8$. His experimental arrangement is also quite distinct from ours and recent studies in that his cylinder does not translate strictly transverse to the flow, and instead moves in an arc. Correspondingly, the arrangement leads to a contribution to the effective restoring force coming from the drag force, thereby leading to nonlinearities in spring stiffness. Despite these obvious basic differences in the experiment, and the fact that there are discrepancies of around $100 \%$ in amplitude when plotted against recent data in the "Griffin" plot, this early data indicates the appearance of an upper branch for sufficiently low damping; see also Figure 6.15 of Naudascher \& Rockwell (1993). Zdravkovich (1990) suggests, in his interesting selective review, that the appearance of a jump to an upper branch in Meier-Windhorst (1939), and the lack of such a jump in other studies (also involving quite different experimental configurations), is a Reynolds number effect, connected with the variation of the vortex formation length with Re. While there is clearly an influence of Reynolds number at low Re values, especially in the laminar shedding regime ( $\mathrm{Re}$ of order 100$)$, our studies here and in Govardhan \& Williamson (1999), for $\mathrm{Re}=3500-10000$, indicate that it is principally the parameter $\left(m^{*} \zeta\right)$ which influences whether the upper branch will appear or not. However, evidence for this deduction at higher Re of order $10^{4}-10^{5}$ would require further experiments.

With respect to the mode transitions, preliminary results in Khalak \& Williamson (1997b) show that the initial $\Leftrightarrow$ upper branch transition is hysteretic, while the upper $\Leftrightarrow$ lower transition involves instead an intermittent switching. It will be shown in the present work that the peak amplitude is governed by the combined parameter $m^{*} \zeta$, over a wide range of $m^{*}$ and $\zeta$, whereas for experiments where $m^{*} \zeta$ is kept constant, we show that the width of the synchronization regime is governed by the mass ratio $m^{*}$. These three modes, and their transitions, will be studied in detail in the present paper. The fact that both an upper and lower branch exist, quite aside from the initial branch, led to two distinct curves of response in the "Griffin" plot, as suggested in the preliminary work of Khalak \& Williamson (1997a), and shown comprehensively in the present paper.

We shall address now the last question posed at the start of Section 1, concerning the definition of "synchronization" or "lock-in", which has apparently led to some recent confusion in the literature. One might consider how the response frequency ratio $f^{*}$ behaves for different mass ratios, as may be seen in Figure 4, taken for reference from Khalak \& Williamson (1997b). The phenomenon of "lock-in" or synchronization [see Blevins (1990), and Sumer and Fredsфe (1997)] means that as the fluid velocity $U^{*}$ is increased, a speed is reached at which the vortex-shedding frequency $\left(f_{\mathrm{O}}\right)$ becomes close to the natural frequency of the structure $\left(f_{N}\right)$, and the two frequencies synchronize. Further increase of $U^{*}$, over a range of synchronization, pulls the shedding frequency away from its nonoscillating value. The shedding frequency and the oscillation frequency $(f)$ remain close to $f_{N}$, and thus the ratio $f^{*}=f / f_{N}$ remains close to unity. This may be seen in Figure 4, for the moderate mass ratios, $m^{*}=10-20$. This classic scenario is approximately correct for large mass, $m^{*}$, but it can be seen that, even in Figure 4, $\mathrm{f}^{*}$ is not precisely equal to unity.

The frequency $f^{*}$, within the regime of significant amplitude response, increases as $m^{*}$ is reduced. This is very clearly seen in the upper plot for $m^{*}=2.4$ where, over the synchronization range $U^{*}=4-11$, the frequency $f^{*}$ rises to $1 \cdot 4$. Initially, we viewed this in Khalak \& Williamson (1997b) as "surprising", but the use of this word is inappropriate, as this phenomenon certainly must be expected, as pointed out clearly in Bearman's (1984) review. The extensive "added mass" coefficients measured by Sarpkaya (1978) would suggest that 

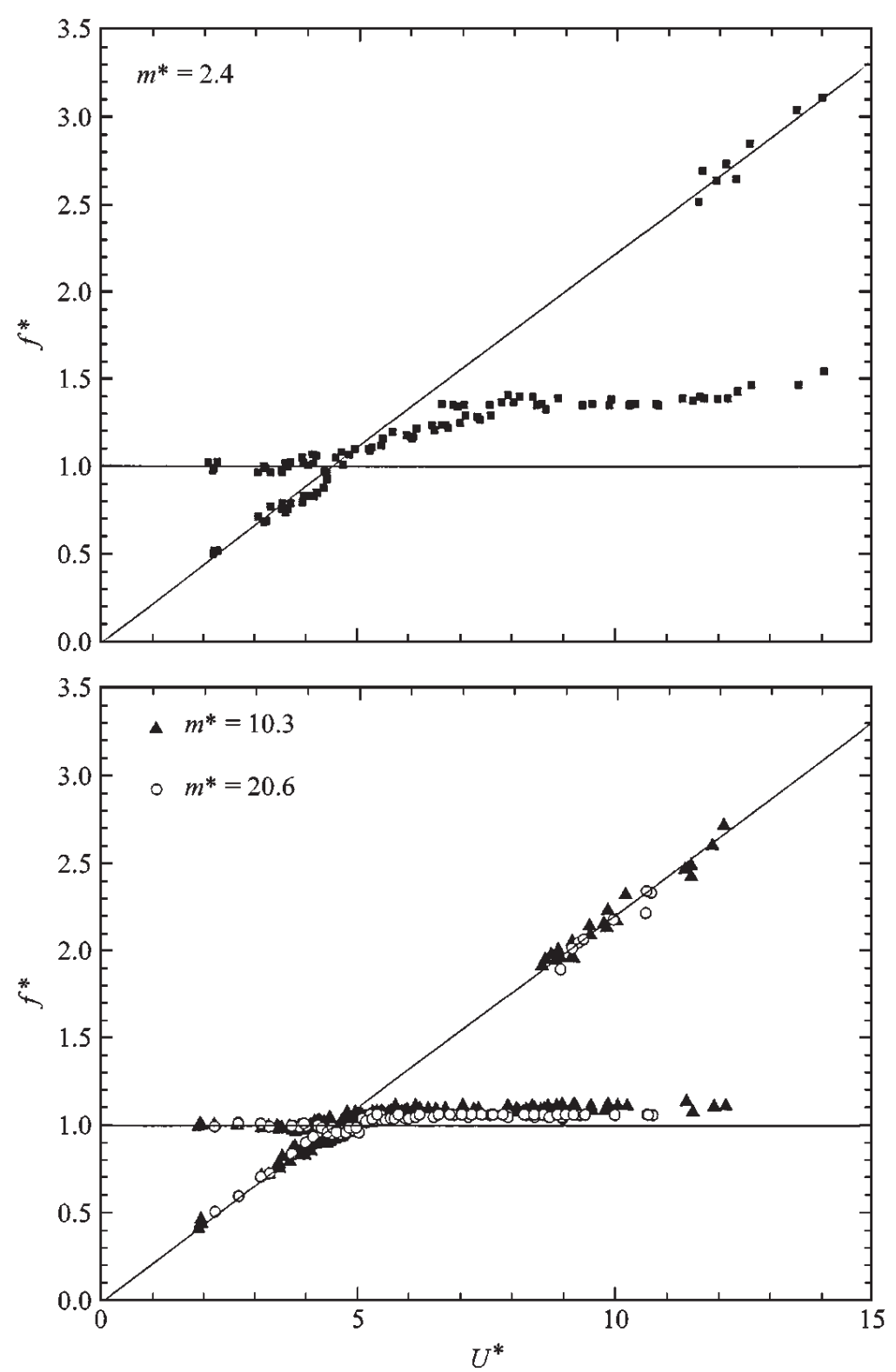

Figure 4. Frequency response for a range of mass ratios, $m^{*}$, through the synchronization regime.

oscillation frequencies at large amplitude would be expected to depart from unity. As mentioned earlier, it is evident by inspection of equation (5), that $f^{*}$ depends on the "effective added mass" coefficent $C_{E A}$, whose significance clearly increases as mass ratio $m^{*}$ becomes smaller. Experimentally, the departure of $f^{*}$ from unity, through the lock-in regime, was shown by Moe \& Wu (1990), and more recently is reported in Khalak \& Williamson (1997b) and in Gharib et al. (1998). We shall return to the question of the departure of $f^{*}$ from unity, later in the present work.

The apparently "nonclassical" behaviour of $f^{*}$, through the synchronization regime, is the result of imposing a low mass ratio. Our experiments for a tethered sphere under vortexinduced vibration at extremely low $m^{*}<1$ (Govardhan \& Williamson 1997) exhibit very similar dynamic phenomena. In fact, the frequency $f^{*}$ exceeds $2 \cdot 0$, which is far from what one might have considered classical lock-in, where $f=f_{N}$, or equivalently $f^{*}=1 \cdot 0$. We cannot thus define lock-in or synchronization such that the shedding frequency is close to the still-fluid $f_{N}$. Nor can it be said that the shedding frequency necessarily matches the 
oscillation frequency, because the wake mode might involve more than two vortices per cycle (as discussed below). We can, however, usefully define synchronization as the matching of the frequency of the periodic wake vortex mode with the body oscillation frequency. Correspondingly, the transverse force frequency must match the oscillation frequency, which is the definition of lock-in now used by Sarpkaya (1995). [In fact, Sarpkaya defines his natural frequency $f_{N}^{\prime}$ to be equal to the body oscillation frequency $f$, such that $f / f_{N}^{\prime}=1$ automatically throughout the lock-in regime. We choose here a fixed $f_{N}$ to serve as a useful reference frequency.] With these considerations in mind, the lock-in, which is reported in Gharib et al. (1998) to be "absent" for small mass ratios, is therefore not actually absent, if we use the definition above and in Sarpkaya (1995).

It is expected that the sharp changes in phase angle through resonance, as velocity $U^{*}$ is increased, will be matched by a switch in the timing of vortex shedding relative to body displacement, and this was shown for the first time by Zdravkovich (1982), using visualizations from previous studies. This has been confirmed by $\mathrm{Gu}$ et al. (1994) from forced vibrations at small $A^{*}=0 \cdot 2$, with further agreement from the simulations of Meneghini \& Bearman (1995) and Lu \& Dalton (1996). Griffin \& Ramberg (1974) showed, for larger-amplitude forced vibration experiments, that an asymmetric mode occurred whereby three vortices form per cycle, as $A^{*}$ increases from 0.5 to $1 \cdot 0$. Subsequent studies over a very wide variation of $A^{*}$ and $U^{*}$ by Williamson $\&$ Roshko (1988), found a selection of vortex formation modes, which were defined as " $2 \mathrm{~S}$ " indicating 2 single vortices shed per cycle, "2P" meaning 2 pairs of vortices per cycle, and the pattern comprising three vortices per cycle thus was termed "P $+\mathrm{S}$ ". Ongoren \& Rockwell (1988) found the equivalent of the $2 \mathrm{P}$ mode, but only for streamwise vibrations, although subsequent DPIV (digital particle image velocimetry) measurements confirm the $2 \mathrm{P}$ mode in forced lateral vibrations (Sheridan et al. 1998), and in forced motions of a tapered cylinder where a "hybrid" mode was found comprising $2 \mathrm{P}$ and $2 \mathrm{~S}$ modes occurring simultaneously along the span (Techet et al. 1998).

The observation in Williamson \& Roshko that forced oscillations corresponding to the initial branch of Feng (1968) formed the $2 \mathrm{~S}$ mode and those on the lower branch formed the 2P mode, has since been confirmed by Brika \& Laneville (1993), who were the first to show visual evidence of these distinct modes in free vibration, and was more recently shown from the DPIV measurements of free vibration modes by Govardhan \& Williamson $(1998,1999)$. The ingenious virtual free vibration experiments by Hover et al. (1998), whereby the mass-spring-damper is simulated in real time on a computer, and where the fluid force is measured, produces a complete response plot which collapses well onto the map of vortex modes from Williamson \& Roshko, again suggesting the initial branch is $2 \mathrm{~S}$ mode, and the lower branch is the $2 \mathrm{P}$ mode. These vortex modes will be shown to be in accordance with observations in the present paper.

In the ensuing sections, following the experimental details in Section 2, we shall present a set of data in the "Griffin" plot in Section 3, which demonstrate that the combined massdamping parameter collapses very well the peak amplitude data, over a wide range of $\left(m^{*}+C_{A}\right) \zeta$. We study the vortex-shedding modes, in brief, and also the collapse of the complete amplitude response plot using the normalized velocity $\left(U^{*} / f^{*}\right)$. In Section 4 , we present lift and drag force measurements, followed by the discussion of response dynamics for the different modes and their transitions in Section 5. Finally, Section 6 comprises the conclusions.

\section{EXPERIMENTAL DETAILS}

We have constructed a custom hydroelastic apparatus, for particular application to very low mass and damping conditions, which operates in conjunction with the Cornell-ONR 


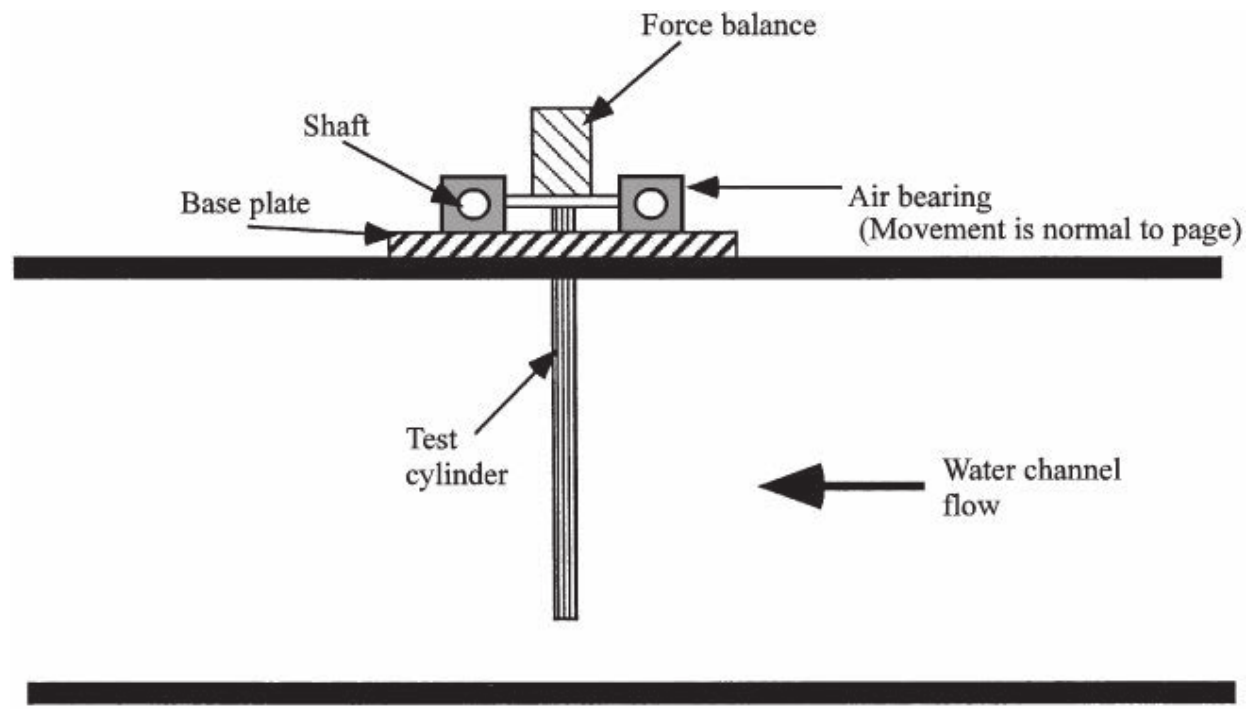

Figure 5. Schematic of the experimental arrangement, seen in side view.

Water Channel. One may refer to Khalak \& Williamson $(1996,1997 b)$ for the details concerning this experimental arrangement, and only the essential outline of the experimental set-up will be mentioned here. An air-bearing facility, allowing only motion transverse to the flow, is located above our water channel, and supports a vertical cylinder in the flow, as shown schematically in Figure 5. A two-axis force balance utilizing LVDTs (linear variable displacement transducers) can measure lift and drag simultaneously with the measurement of displacement, with the latter being measured using a noncontact (magnetostrictive) position transducer. The inertial force was subtracted from the total measured transverse force by post-processing of the data. These pieces of equipment are part of the overall oscillating mechanism above the water surface. Two test cylinders are employed of diameters 1.5 and 2.0 in, and we use length-diameter ratios of 10 and 8.5 , respectively. Our set-up has an order of magnitude lower mass-damping parameter $m^{*} \zeta$ than most of the previous experiments of this type, as shown in Khalak \& Williamson (1996).

\section{AMPLITUDE RESPONSE, WAKE MODES AND THE "GRIFFIN" PLOT}

In this section, we shall study the individual effect of varying the mass ratio, $m^{*}$, while keeping the mass-damping parameter $m^{*} \zeta$ at a constant very low value. The dissimilar response plots will be collapsed onto a single response curve by a suitable normalization of the velocity. We shall also observe briefly the synchronized vortex wake modes associated with each amplitude branch of response. Finally, we shall present peak-amplitude data in the form of a "Griffin" plot, showing a good collapse of data along only two curves, for a wide variation of mass ratios, $m^{*}$. Our experimental data is not well matched by the standard empirical curves, that have been traditionally employed in the Griffin plot, over the past 20 years, to represent accumulated data from previous VIV experiments.

The complete response plots of $A^{*}$ versus normalized velocity $U^{*}$ are found in Figure 6(a), where, although the mass ratio $m^{*}$ is individually varied over a large range $\left(m^{*}=1-20\right)$, the mass-damping parameter $\left(m^{*} \zeta\right)$ is kept approximately at a constant level $(0 \cdot 14-0 \cdot 19)$. Although the peak amplitudes in the upper and lower branches appear to remain the same, the effect of smaller $m^{*}$ leads to an extended synchronization regime. In fact these, and a great many further response plots in Govardhan \& Williamson (1999), support the fact that for constant $m^{*} \zeta$, the peak amplitude remains constant, irrespective of the individual 

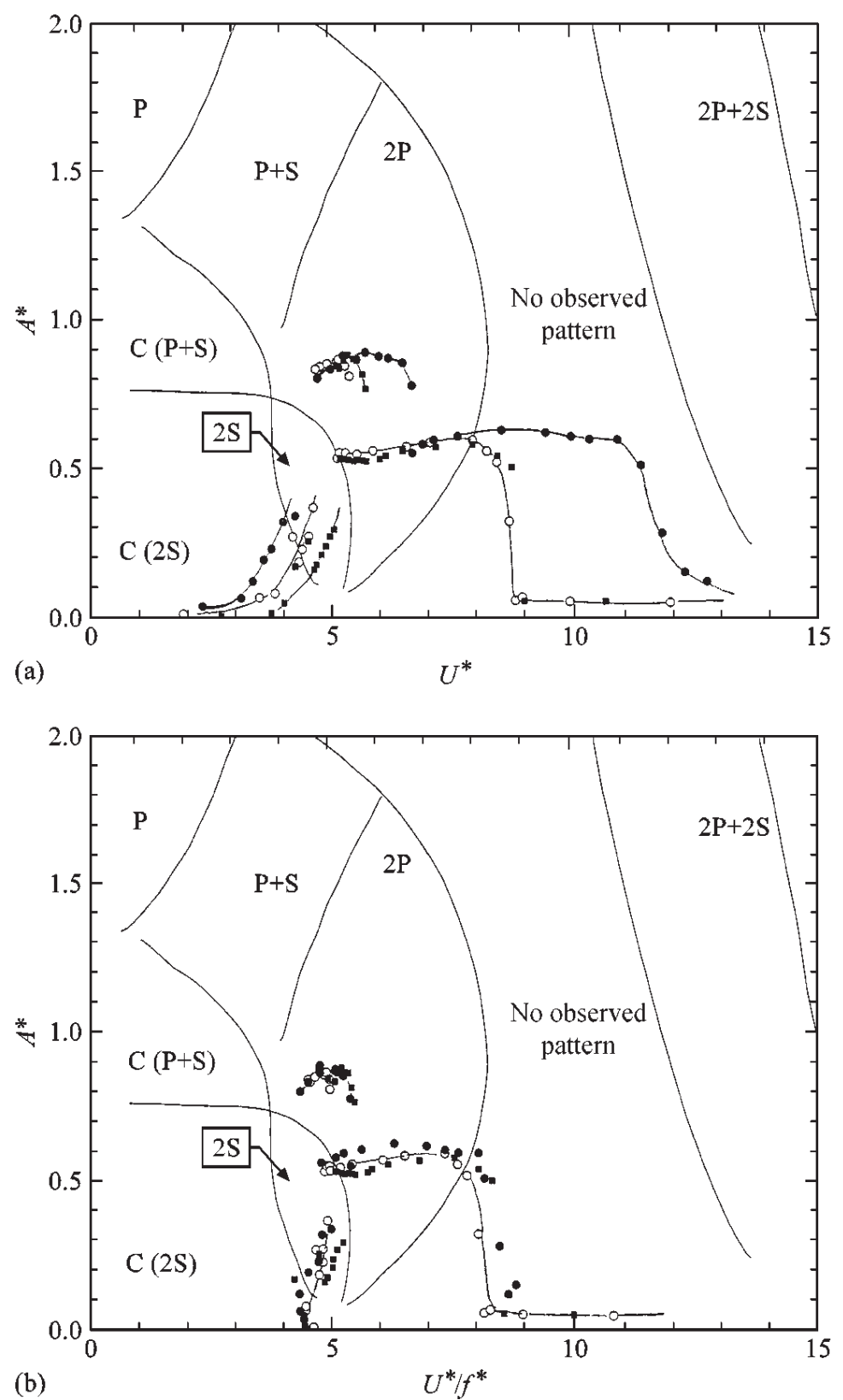

Figure 6. Amplitude response for constant $m^{*} \zeta$, but for different values of mass ratio, $m^{*}$, all plotted in the Williamson-Roshko (1988) map of wake pattern regimes. A beautiful collapse of these response plots emerges when we normalize velocity by $U^{*} / f^{*}$, rather than $U^{*}$ alone. $\left[U^{*} / f^{*}\right.$ is equivalent to "true" reduced velocity in Hover et al. (1998) and Moe and Wu (1990).] This appears to be the first demonstration of such a collapse of data from free vibration experiments. Note that "No observed pattern" means that no wake vortex pattern was observed which had the same period as the oscillation. Data: $\left(\cdot, m^{*}=2.4\right.$ and $m^{*} \zeta=0 \cdot 014 ; \bigcirc, m^{*}=10 \cdot 3$ and $m^{*} \zeta=0 \cdot 017 ; \mathbf{\square}, m^{*}=20.6$ and $m^{*} \zeta=0 \cdot 019$.

values of mass $m^{*}$ and damping $\zeta$, at least within the large range of mass and damping that has been studied in these works.

By plotting the amplitude data in Figure 6(a) over the top of the Williamson-Roshko (1988) map of different wake modes, from their forced vibration experiments, it is apparent that the data for the lowest mass ratio $m^{*}=2 \cdot 4$ does not follow the expected drop-off in amplitude when $U^{*}$ exceeds the limit of the periodic $2 \mathrm{P}$ mode, and moves into the regime of desynchronization. The high- $m^{*}$ amplitudes, however, sharply diminish when the vortex wake pattern becomes desynchronized. It is clear that for very low mass ratios, the 
conventional normalized velocity $U^{*}$ is not perhaps the best parameter with which to make a response plot.

A useful approach adopted by those numerically simulating forced oscillations is to vary the forcing frequency relative to the (nonoscillating) vortex-shedding frequency $\left(f_{v o}\right)$ at the relevant Reynolds number, giving the parameter $\left(f / f_{v o}\right)$. The inverse of this can be written in terms of our nondimensional groups here as

$$
\frac{f_{V O}}{f}=\left(\frac{f_{V O} D}{U}\right)\left(\frac{U}{f D}\right)=S\left(\frac{U^{*}}{f^{*}}\right) .
$$

In our case, the Strouhal number, $S$, is closely constant over all these experiments, and we choose to present the amplitude data $A^{*}$ as a function of $U^{*} / f^{*}$ :

$$
\left(\frac{U^{*}}{f^{*}}\right)=\left(\frac{U}{f D}\right) \text {. }
$$

In other words, the wake patterns will be expected to be the same when one matches both the amplitude $A^{*}$ and also the velocity normalized by the actual oscillating frequency $(f)$. The ensuing normalization in Figure $6(\mathrm{~b})$ collapses the data beautifully. This appears to be the first time that such a collapse of data has been shown from free vibration experiments.

It should be mentioned, however, that a similar normalization has been used before, not only in the numerical simulations, but also in experiment. Moe \& Wu (1990) and Hover et al. (1998) have used the terminology "true" reduced velocity to refer to U/fD as in equation (14), and they use "nominal" reduced velocity to refer to $U / f_{N} D$, or $U^{*}$ in the present terminology. Sarpkaya (1995) uses his parameter $V_{r} \mathrm{St}$, equal to $\left(U / f_{N}^{\prime} D\right) /(f D / U)$, which is the same as $f_{\mathrm{vo}} / f_{N}^{\prime}$. This is equivalent to $S(U / f D)$, so long as one remembers that what he calls his "natural" frequency $f_{N}^{\prime}$ is not a fixed value, but is the actual oscillating frequency.

Although it may appear that the normalized velocity $\left(U^{*} / f^{*}\right)$ necessarily collapses any set of amplitude data for a variation of $m^{*}$, so long as $m^{*} \zeta$ is held constant, this is not known a priori since $A^{*}$ depends on the oscillation frequency $f^{*}$ [see equation (4)]. In order to demonstrate that the parameter $U^{*} / f^{*}$ would necessarily collapse the amplitude response with respect to variations in $m^{*}$, one would have to show that $A^{*}$ is unique for a given $U^{*} / f^{*}$, at constant $\left(m^{*}+C_{A}\right) \zeta$; it turns out that this is not the case, which is explained as follows. In our present case, at a given $U^{*} / f^{*}$, we can ascribe the following dependencies for the magnitude of the fluid force $C_{y} \sin \phi$ and the oscillation frequency $f^{*}$ :

$$
\begin{aligned}
& C_{y} \sin \phi=\text { function }\left\{A^{*}\right\}, \\
& f^{*}=\text { function }\left\{A^{*}, \mathrm{~m}^{*}\right\} .
\end{aligned}
$$

Imposing the condition that $\left(m^{*}+C_{A}\right) \zeta$ and $U^{*} / f^{*}$ are constant, and substituting these dependencies into equation (4) produces the result that

$$
A^{*}=\text { function }\left\{m^{*}\right\} \text { only. }
$$

It is thus not to be expected in general that simply replotting the complete amplitude response as a function of $U^{*} / f^{*}$ will yield a collapse of the data. The collapse happens to work well for small $m^{*} \zeta$. This is because the upper branch frequency $f^{*} \approx 1.0$ for all three values of $m^{*}$ used here, giving the same peak amplitudes; and the lower branch amplitudes are closely the same also, because an amplitude saturation level, independant of $m^{*} \zeta$, has been reached for low- $\left(m^{*} \zeta\right)$; (see the Griffin plot, in Figures 8 and 9). 

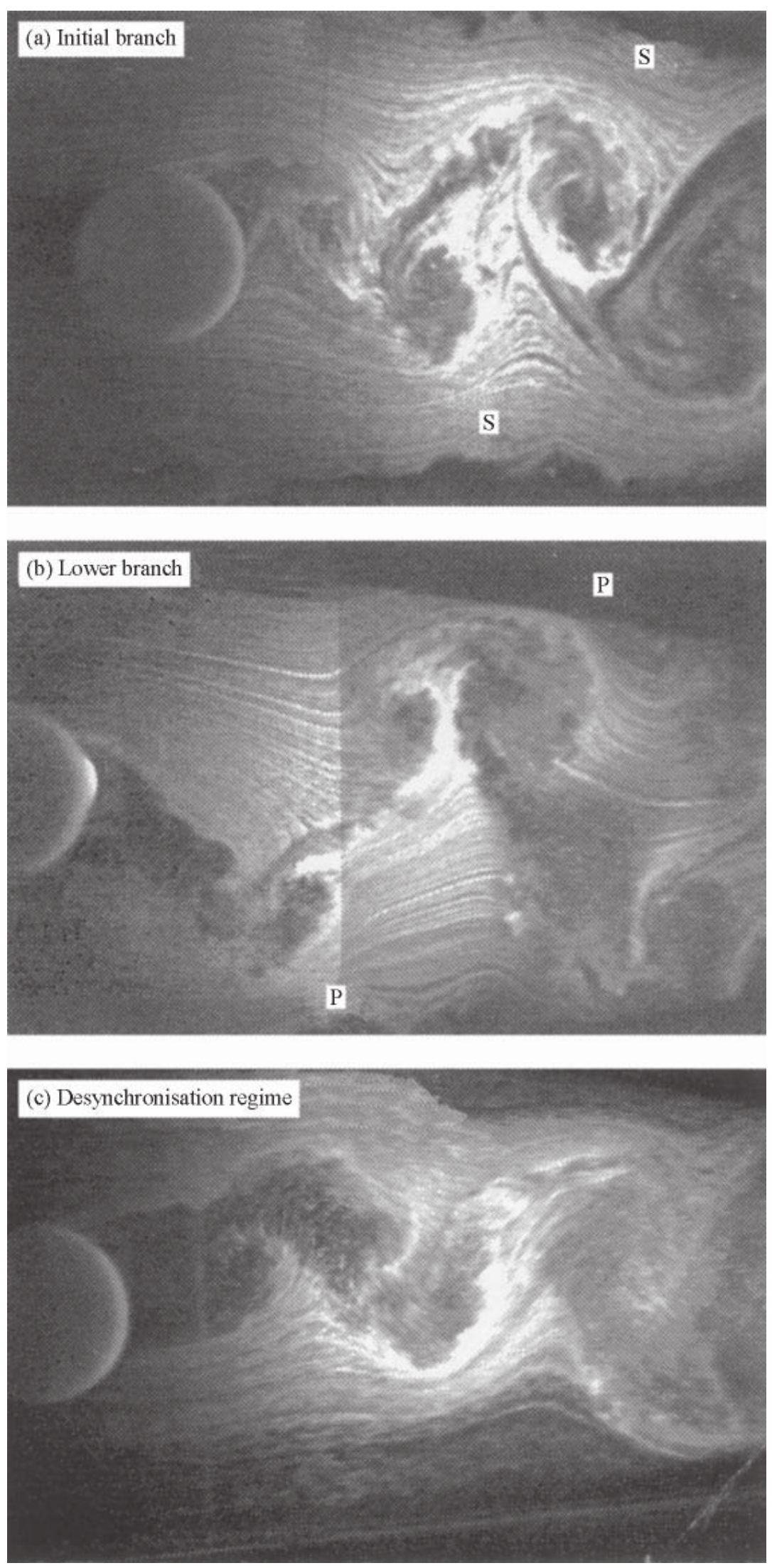

Figure 7. Hydrogen-bubble flow visualization of the initial branch ( $2 \mathrm{~S}$ mode), the lower branch ( $2 \mathrm{P}$ mode), and the Desynchronized mode. (a) $\operatorname{Re}=4500$; (b) $\operatorname{Re}=6000$; (C) $\mathrm{Re}=9000$. 
The amplitude branches are found to correspond with different synchronized vortex wake patterns, as shown in Figure 7. The initial branch in the amplitude response appears to lie in the $2 S$ mode of the Williamson-Roshko map of wake patterns, meaning two single vortices formed per cycle, and this is supported by the hydrogen bubble visualization in Figure 7(a). The lower branch seems to be in the $2 P$ mode regime, and this corresponds well with the visualization in (b), indicating two pairs of vortices being formed per cycle. This supports the scenario put forward, from forced oscillation experiments, in Williamson and Roshko (1988). The visualizations in Figure 7 are in accordance with Brika \& Laneville (1993) for their cable vibrations, who were the first to confirm that the $2 \mathrm{~S} \Leftrightarrow 2 \mathrm{P}$ mode transition corresponds with the initial $\Leftrightarrow$ lower branch of body dynamics, in free vibrations. Further visualization of the upper branch appeared to be the $2 \mathrm{~S}$ mode as in (a), although it is not evident from this somewhat unclear visualization technique whether it could be distinct from the initial branch. Despite the observations made here, the somewhat unclear dye or bubble visualizations that are possible at these Re of order $10^{3}-10^{4}$ should not be used as firm proof of the vortex dynamics modes. Subsequent DPIV vorticity measurements to be found in Govardhan \& Williamson (1999) clarify firmly the vortex dynamics associated with the different amplitude branches, including the upper branch.

Finally, in this section, we look at the Griffin plot of peak-amplitude data. An important question that has been debated for about 20 years is whether a combined mass-damping parameter $m^{*} \zeta$ could reasonably collapse peak amplitude data $A_{\max }^{*}$ in what we denote here the "Griffin" plot. On the one hand, Sarpkaya $(1978,1995)$ has firmly stated that the response is governed by $m^{*}$ and $\zeta$ independently, and not by the combined mass-damping $m^{*} \zeta$. Zdravkovich also states that a combined $m^{*} \zeta$ paramater is not legitimate for $m^{*}<10$. On the other hand, Naudascher \& Rockwell (1993) suggest that even down to low $m^{*} \zeta$, the Griffin plot does quite well to collapse $A_{\max }^{*}$ data. However, as can be seen in the Griffin plot of Figure 1, when the vertical axis for $A^{*}$ is plotted as a linear scale (rather than by the conventional logarithmic scale), the scatter in the data is evidently large.

Previous scattered results, in Figure 1, would lead one to deduce that the Griffin plot does not collapse peak-amplitude data, but one must note that all these data are from markedly different experimental set-ups, and there also will exist differences in measurement accuracy and procedure. In the present study, we set out to plot $A_{\max }^{*}$ versus $\left(m^{*}+C_{A}\right) \zeta$ in a Griffin diagram in Figure 8, for a wide variation of mass ratios, $m^{*}$, and for a uniformly similar set of experiments. Only in this fashion, might one expect to accurately question whether the amplitude data may be collapsed in the Griffin plot. We choose to plot the peak-amplitude data both for the Upper branch and also for the Lower branch in Figure 8.

The collapse of the data appears to be very good; the data sets lie along only two distinct curves, one curve for the upper branch and one for the lower branch. In fact, the Griffin plot appears to give a clear functional relationship between $A_{\max }^{*}$ and $\left(m^{*}+C_{A}\right) \zeta$ over a wide range of parameters; for $m^{*}>2$, and for $\left(m^{*}+C_{A}\right) \zeta>0 \cdot 006$. The use of the Griffin plot to collapse peak-amplitude data versus the mass-damping is confirmed over a much larger regime of $\left(m^{*}+C_{A}\right) \zeta$ than could be validated by the previous scattered data, and is a regime that extends to two orders of magnitude lower $\left(m^{*}+C_{A}\right) \zeta$ than proposed by Sarpkaya, and often quoted in the literature, namely $S_{G}>1$, or equivalently $\left(m^{*}+C_{A}\right) \zeta>0 \cdot 40$.

It was indicated in Section 1 that the peak response $A_{\max }^{*}$ is a function of $m^{*}$, as well as of $\left(m^{*}+C_{A}\right) \zeta$. The influence of $m^{*}$ comes from the frequency $f^{*}$ in equation (4), but it is to be noted that, in determining the maximum amplitude throughout the present work, it turns out that $f^{*} \approx 1 \cdot 0$, and therefore $A_{\mathrm{MAX}}^{*}$ is a function only of $\left(m^{*}+C_{A}\right) \zeta$, to good approximation. However, it must be expected that there will be limits when the independent value of $m^{*}$ will influence the peak response, although the limits set by Sarpkaya seem to be 


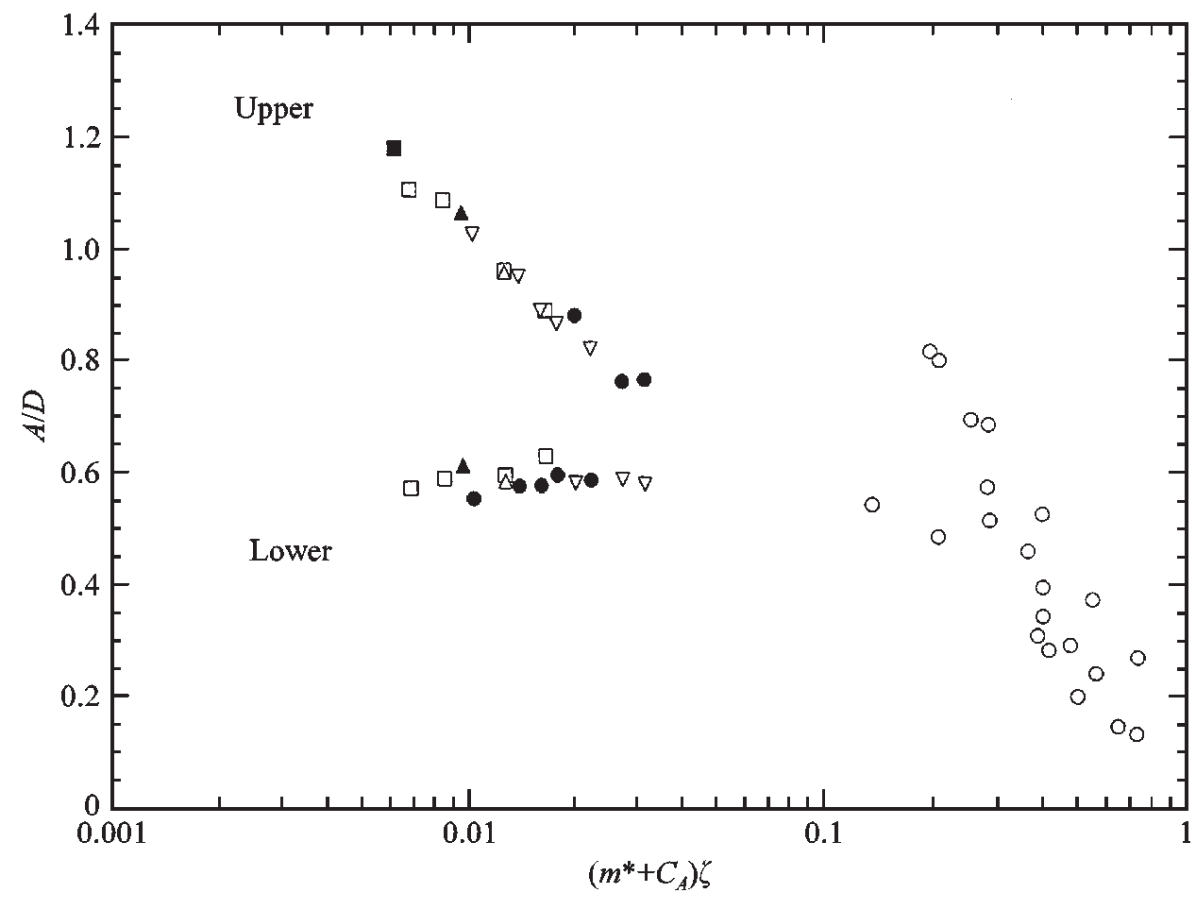

Figure 8. "Griffin" plot of the upper and lower branch peak-amplitude data versus $\left(m^{*}+C_{A}\right) \zeta$, for a wide variety of mass ratios, $m^{*}$. The data lies cleanly along only two curves in this plot. $\mathbf{\square}, m^{*}=1 \cdot 2 ; \square, m^{*}=2 \cdot 4 ; \boldsymbol{\Delta}$ $m^{*}=3 \cdot 3 ; \triangle, m^{*}=10 \cdot 1 ; \nabla, m^{*}=10 \cdot 3 ; \boldsymbol{\bullet}, m^{*}=20 \cdot 6 ; \bigcirc$, data compiled by Griffin (1980).

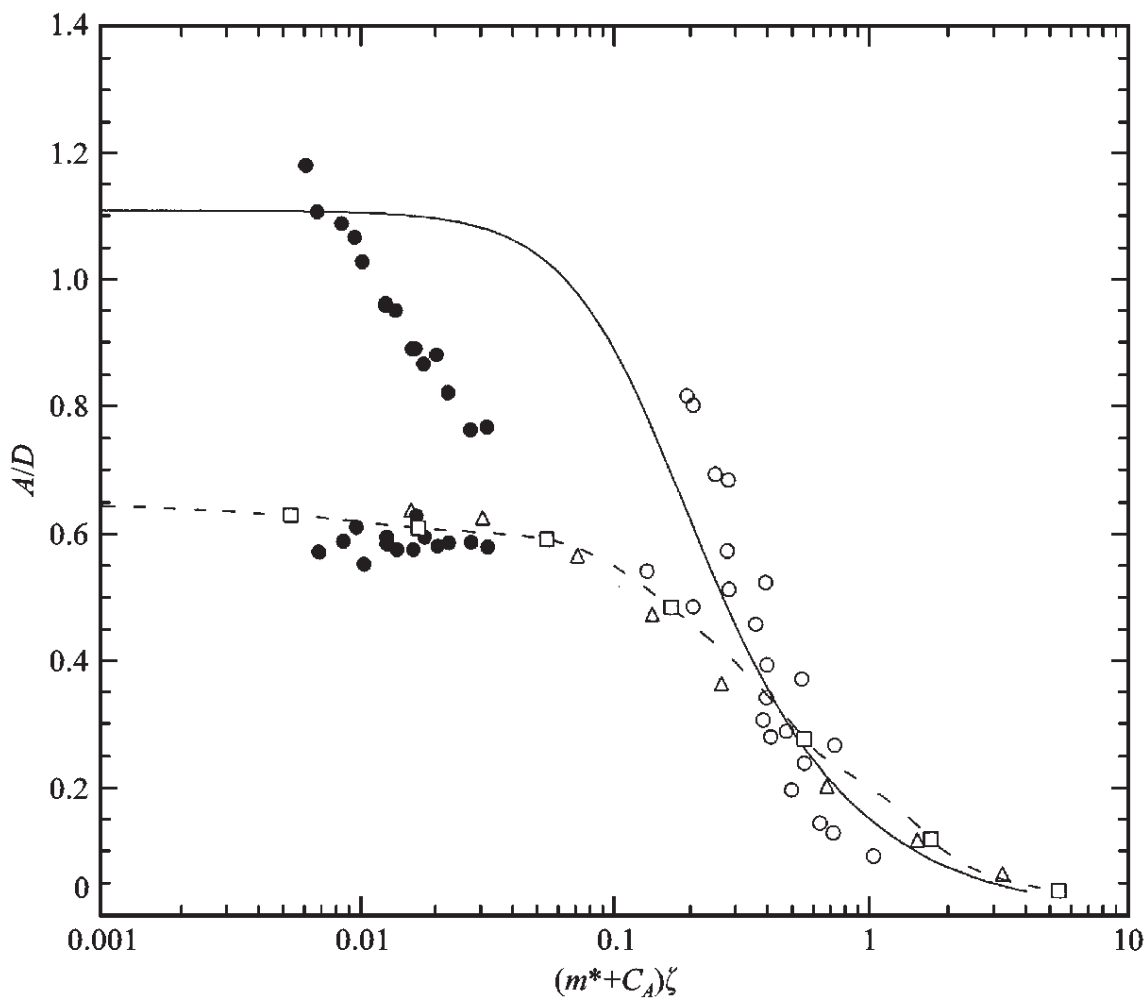

Figure 9. "Griffin" plot of the present peak amplitude data, compared with data compiled by Griffin (1980), with DNS computations, and with a classical curve fit by Skop and Balasubramanian (1997), as used also in Figure 1. This demonstrates that the present experimental data does not appear to follow the classical curve fits previously used. ๑, Present data; $\bigcirc$, data compiled by Griffin (1980), __, Skop \& Balasubramanian (1997); $\triangle$, Blackburn \& Karniadakis (1993); -- $\square--$, Newman \& Karniadakis (1996). 
somewhat restrictive. Further ongoing measurements are being conducted to probe the limits within which $\left(m^{*}+C_{A}\right) \zeta$ remains a valid parameter to predict peak response $A_{\max }^{*}$.

We now compare the peak-amplitude data with direct numerical simulation results in the Griffin plot of Figure 9. The extensive two-dimensional simulation data from Blackburn \& Karniadakis (1993) and by Newman \& Karniadakis (1996), for the low Reynolds number of 200 and 100 respectively, are shown in this plot. It is immediately apparent that the maximum amplitudes coming from the simulations lie close to the lower branch, $A^{*} \sim 0.6$. Coupled with the fact that the low-Re experiments (Anagnostopoulos \& Bearman 1992) also do not exhibit an upper branch, one might deduce that there are differences in the vortex dynamics at low Reynolds numbers, which prohibit the jump to an upper branch. Evidence for this also comes from Williamson \& Roshko (1988), who found only the P $+\mathrm{S}$ mode of vortex formation, in place of the whole 2P-mode region in Figure 6, when the Reynolds number was below about 300. In fact, the classical smoke visualizations of Griffin \& Ramberg (1974) demonstrate only the $\mathrm{P}+\mathrm{S}$ mode for $\mathrm{Re}<190$, and they never observed the $2 \mathrm{P}$ mode in their laminar-regime studies.

One might then ask what are the vortex dynamics found in the low-Re simulations. For the whole synchronization regime, corresponding to $A^{*}<0 \cdot 6$, several simulations for free vibration find only the $2 \mathrm{~S}$ mode throughout the lock-in regime (Fujarra et al. 1998; Newman $\&$ Karniadakis 1996). (This shows that, although the maximum amplitudes from the simulations lie closely along the lower branch experimental data, one should not then infer that the DNS results correspond to a lower branch or to the $2 \mathrm{P}$ mode found in the higher-Re experiments.) On the other hand, in low-Re simulations of forced oscillations, either for higher amplitudes roughly $A^{*}>0 \cdot 6$, or for frequencies below a critical value, only the $\mathrm{P}+\mathrm{S}$ mode has been found (Meneghini \& Bearman 1995; Blackburn \& Henderson 1995). One may conclude that the absence of the upper branch in the simulations is due to the fact that the $\mathrm{P}+\mathrm{S}$ mode does not give a net energy transfer from fluid to body motion over a cycle, unlike the $2 \mathrm{P}$ mode at higher $\mathrm{Re}$. It remains to decisively prove this point with free and forced vibration studies at low-Re, a study which we are pursuing.

Finally, the data in Figure 9 is also compared with the curve fit adapted from Skop \& Balasubramanian (1997), using the functional relationship derived by Sarpkaya (1978), as in equation (11). It seems clear that our experimental data here does not follow such a functional representation. Even for the smallest $\left(m^{*}+C_{A}\right) \zeta \sim 0.006$, the peak amplitudes are not close to saturating to a specific value, as they do in the functional curve. Further intense work is being conducted to answer what is perhaps the most fundamental question in this type of study: What is the limit of peak amplitude as $\left(m^{*}+C_{A}\right) \zeta$ gets ever smaller? In fact, our largest peak amplitude, $A^{*}=1 \cdot 20$, has already exceeded the largest value found in any previous experiment $\left(A_{\max }^{*} \sim 1 \cdot 10\right)$, and yet it remains steadily increasing, with no signs of amplitude saturation, as mass-damping is diminished.

\section{LIFT AND DRAG FORCE}

Despite the extensive force measurements for a cylinder undergoing transverse forced vibration [see, for example, Sarpkaya (1978), Staubli (1983), Gopalkrishnan (1993), and others], there have appeared almost no direct lift force measurements in the literature for such an elastically mounted arrangement, with the exception of Khalak \& Williamson (1997b). One should note, however, that Sarpkaya (1995) recently presented a set of drag measurements for a cylinder which can oscillate both in-line and transverse to the flow. Such a two-degree-of-freedom arrangement, as pioneered by Sarpkaya, should yield useful new results, and several groups are now working on this problem. We should also note that Hover et al. $(1997,1998)$, in conjunction with the research group of Michael S. Triantafyllou 

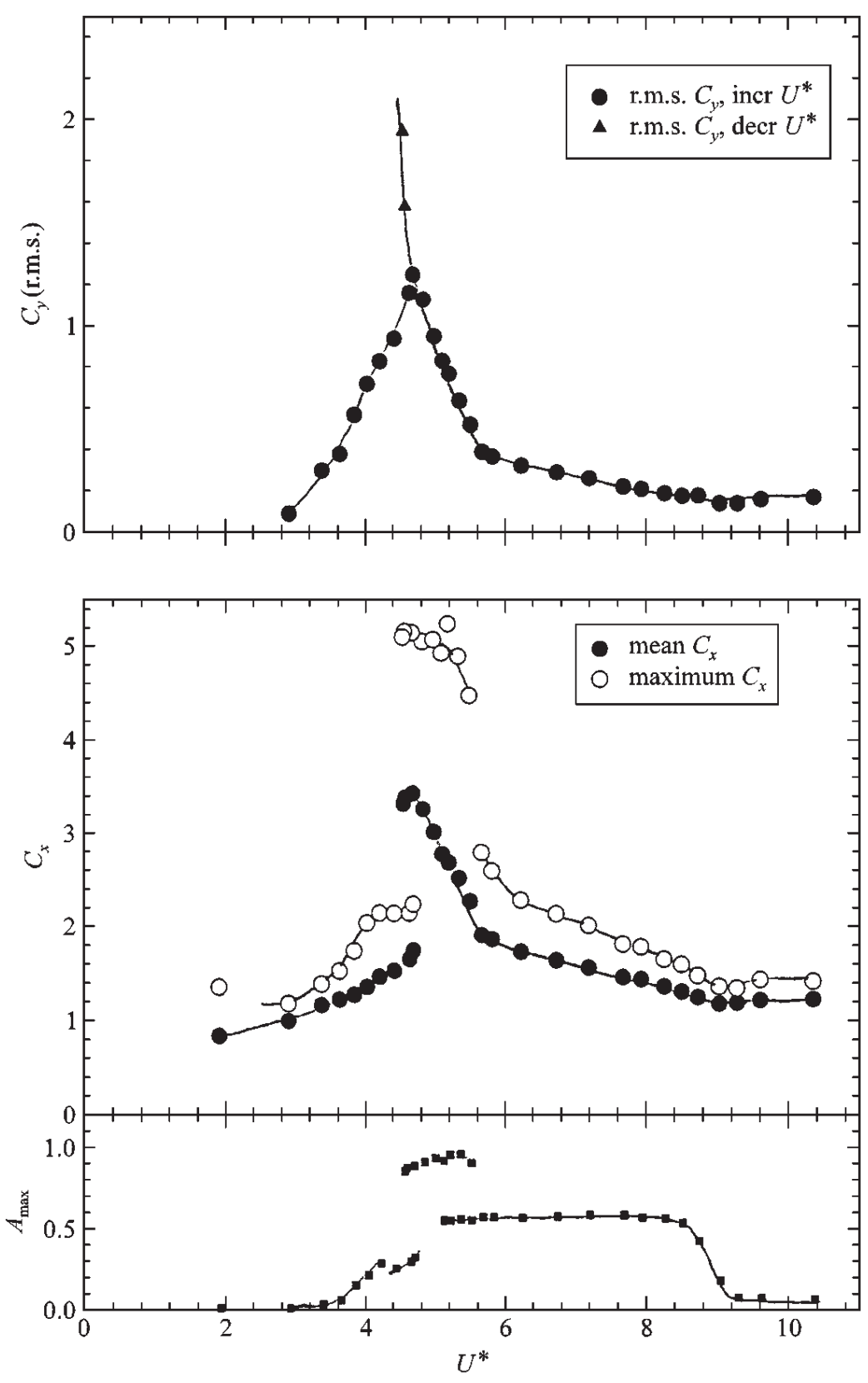

Figure 10. Variation of lift and drag force coefficients versus normalized velocity, $U^{*}$, for $m^{*}=10 \cdot 1$. In the lift force plot, the triangular symbols represent lift force only found for decreasing velocity $U^{*}$, illustrating the presence of a hysteresis.

at MIT, have developed an ingenious experiment, namely an equivalent free-vibration arrangement, whereby a cylinder is made to oscillate as if it were free, by simulating the mass-spring-damper in real time on a computer, and by using also as input the instantaneous force measured on the cylinder. In the present work, we shall briefly present force measurements of lift and drag for a hydroelastic cylinder of very low mass and damping. We shall also present a set of plots which show how the maximum, the mean, and the fluctuating forces are magnified by the body oscillations, as a function of amplitude, $A^{*}$.

Force measurements were made for $m^{*}=10 \cdot 1$ and $\zeta=0 \cdot 00134$, as shown in Figure 10. The drag forces measured during such a free-vibration experiment are massive, reaching 
a maximum $C_{X(\max )}$ of $5 \cdot 2$, with mean $C_{X(\text { mean })}$ of around $3 \cdot 5$. This is a three-fold increase over the stationary case (denoted as $C_{X \mathrm{O}}$ ), but the difference between the maximum and mean drag values also indicates the large drag force fluctuations, which we mention further below. The lift force $C_{Y(\mathrm{rms})}$ reaches $2 \cdot 0$, which corresponds to a maximum $C_{Y}$ near 3 , suggesting a five-fold increase in lift force over the stationary cylinder case. As we increase $U^{*}$, both the drag and lift rapidly increase through the initial excitation regime, they peak at the beginning of the upper branch region, they decrease rapidly throughout the upper branch, and then gradually diminish through the lower branch region. The most striking feature of the r.m.s. lift plot is the very sharp peak, which occurs right at the transition between initial excitation region and the upper branch. Before the transition, the lift is rapidly increasing, while after the transition in the different mode of response, the lift is rapidly decreasing, thus yielding our very sharp lift peak. Surprisingly, the lift in the lower branch is very low, even smaller than the stationary case at equivalent Reynolds numbers (around 6000).

\subsection{Amplification of Forces due to Vibration}

The forced vibration of a structure can yield larger fluid-induced forces than for fixed cylinders, a result which is well-known from past works referenced in the reviews mentioned earlier, although the extent of the increase is certainly very large. In the case of biharmonic free vibrations, involving simultaneous oscillations in-line and transverse to the flow, Sarpkaya (1995) finds that the mean drag for an oscillating cylinder can become 3.5 times that for the static cylinder, which is comparable to values found here. He also gives a mean drag around three times the static case, if the cylinder is forced into transverse vibration (Sarpkaya 1978). The recent work of Hover et al. (1998), using their equivalent freevibration experiment, gives a r.m.s. lift coefficient of roughly $1 \cdot 6-2 \cdot 0$, when the mass-damping is set to zero $\left(m^{*} \zeta=0\right)$, which is comparable to the present values (we computed their value from their power spectral density of the signal, taken near the lift frequency).

Magnification of mean and maximum drag force, relative to fixed cylinder values, as a function of amplitude $A^{*}$, are shown in Figure 11. (The two data points from the present study correspond to $m^{*}=3.3$ and 10.1.) Although the magnification of $C_{X(\max )}$ is not apparently linear with $A^{*}$, the magnification of $C_{X(\text { mean })}$ is roughly linear, as found originally by Sarpkaya (1978). He found that his forced-oscillation drag data followed the formula

$$
\left(\frac{C_{X(\text { mean })}}{C_{X O(\text { mean })}}\right)=1+2\left(\frac{A}{D}\right)
$$

which suggests that the mean drag is approximately proportional to the apparent projected area mapped out by the oscillating body. This is supported by the present free-oscillation data.

We therefore find, at least in our experiments to-date, a five-fold increase in the maximum drag, and a seven-fold increase in the r.m.s. lift force (see Figure 11(d)), but perhaps the most impact is given by considering the increase in fluctuating drag (see Figure 11(c)). For a static cylinder at Reynolds number of 10000, Gopalkrishnan (1993) gives a fluctuating drag which is around $2 \%$ of its mean value. For our $m^{*}=3.3$ case above, the fluctuating drag is around $60 \%$ of the mean value, which gives a fluctuating drag coefficient of $2 \cdot 2$, around 102 times what is measured for the stationary cylinder case! This compares well with the typical fluctuating drag coefficients (2.0) measured from forced oscillation experiments by Gopalkrishnan (1993), for $A / D \sim 0 \cdot 75$, and at the same normalized frequencies. 

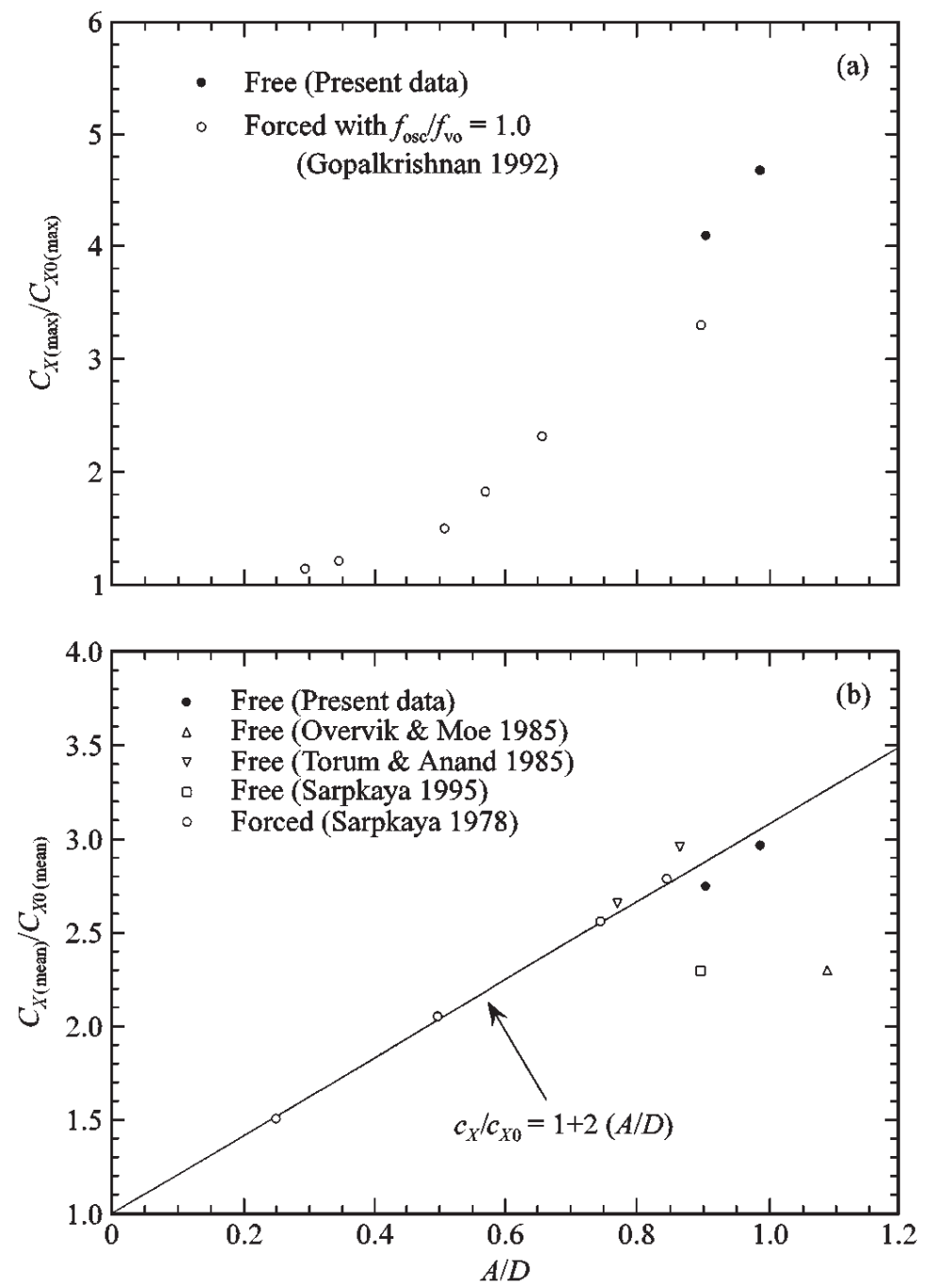

Figure 11. (a) Amplification of maximum drag force and (b) mean drag force, versus amplitude $A^{*}$, in free vibration.

\subsection{Derivation of Force by using Response Data (A* and $f *$ )}

In our studies of vortex-induced vibration of a sphere (Govardhan \& Williamson 1999), it has been found difficult to measure the fluctuating lift force directly, although it is simple to estimate the lift force using displacement response data $\left(A^{*}\right.$ and $\left.f^{*}\right)$, using an assumed equation of motion. It is possible to determine the conditions when such a simple approach is useful, in the present study with the cylinder, by comparing estimated forces with the directly measured forces. We set $\left\{U^{*}, m^{*}, \zeta\right\}$, and measure $\left\{A^{*}, f^{*}\right\}$. We can then find $\left(C_{Y} \sin \phi\right)$ and $\left(C_{Y} \cos \phi\right)$ from a rearrangement of equations (4) and (5):

$$
\begin{gathered}
C_{Y} \sin \phi=\frac{4 \pi^{3} A^{*}\left(m^{*}+C_{A}\right) \zeta}{\left(U^{*} / f^{*}\right)^{2} f^{*}} \\
C_{Y} \cos \phi=\frac{2 \pi^{3} A^{*}}{\left(U^{*} / f^{*}\right)^{2}} C_{E A}
\end{gathered}
$$

We can solve for the two unknowns $\left\{C_{Y}, \phi\right\}$ since all the other variables are known:

$$
C_{Y}=\sqrt{\left(C_{Y} \sin \phi\right)^{2}+\left(C_{Y} \cos \phi\right)^{2}},
$$



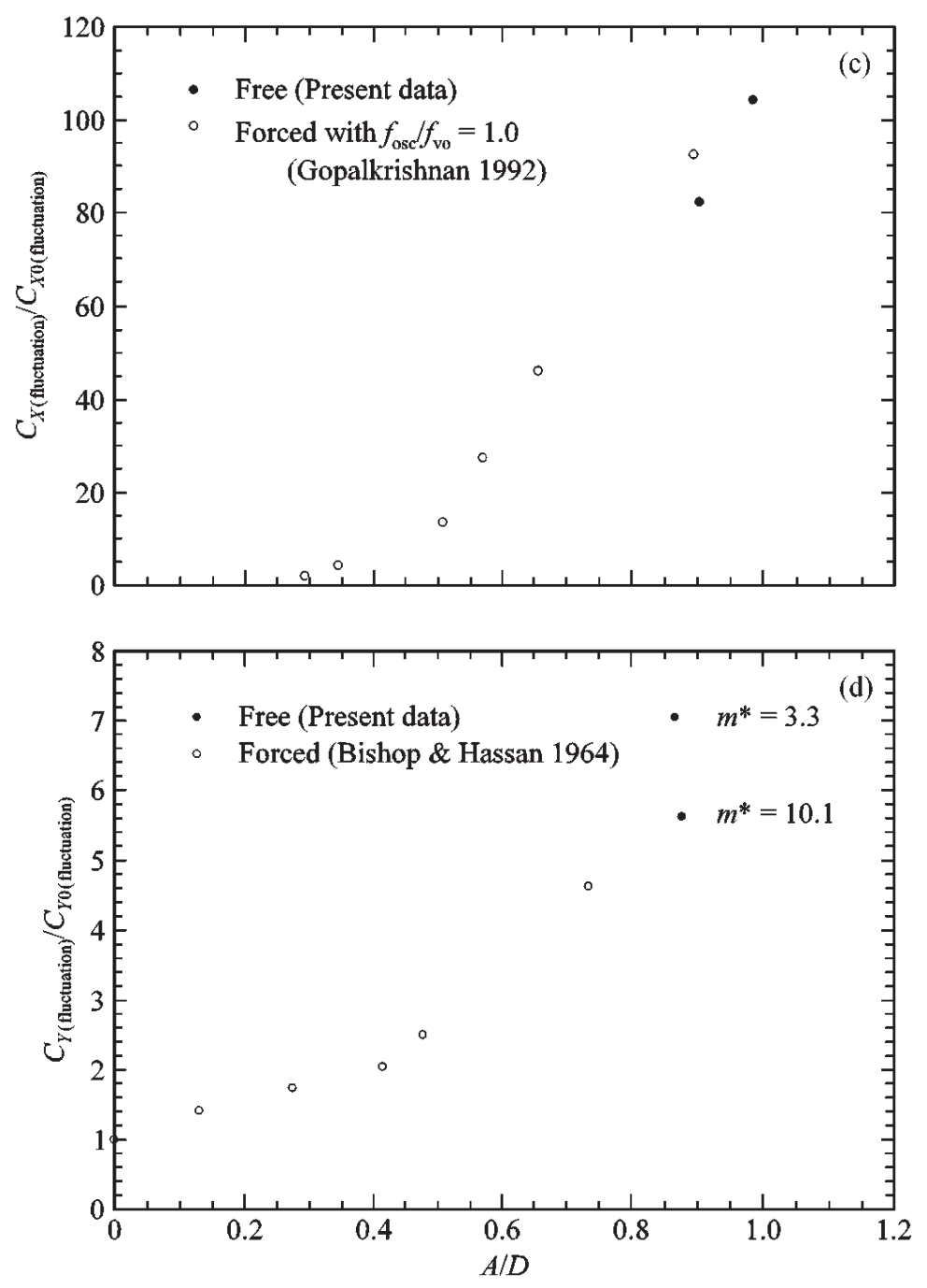

Figure 11. (c) Amplification of fluctuating drag force and (d) fluctuating lift force

$$
\phi=\tan ^{-1}\left(\frac{C_{Y} \sin \phi}{C_{Y} \cos \phi}\right)
$$

In Figure 12, we compare these simple estimates of force with directly measured data, for two values of $m^{*}=3 \cdot 3$ and $10 \cdot 1$, including only $C_{Y}$ here for brevity. Interestingly, the lower value of $m^{*}=3 \cdot 3$ gives by far the better estimate of $C_{Y}$. The agreement between the data is especially good where the response data was found to be most periodic, in the lower branch.

In the case of the high mass ratio of $m^{*}=10 \cdot 1$, the agreement is not good, and this is primarily because of the difficulty in measuring the frequency $f^{*}$ to sufficient resolution. It may be shown that for high $m^{*}$, the effective added mass, $C_{E A} \sim 2 m^{*}\left(1-f^{*}\right)$. The errors in determining $C_{E A}$ are large, because the resolution of measuring frequency $\left( \pm \Delta f^{*}\right)$ becomes very important:

$$
\left(\frac{\Delta C_{E A}}{C_{E A}}\right)=\left(\frac{\Delta f^{*}}{f^{*}-1}\right)
$$

One should note that for large $m^{*}, f^{*}$ is very close to $1 \cdot 0$, so that $\Delta f^{*}$ can become comparable or even larger than $\left(f^{*}-1\right) . C_{E A}$ influences the $C_{Y} \cos \phi$ term, and for very small $\zeta$, the $C_{Y} \cos \phi$ term in equation (19) becomes dominant, therefore large errors in 

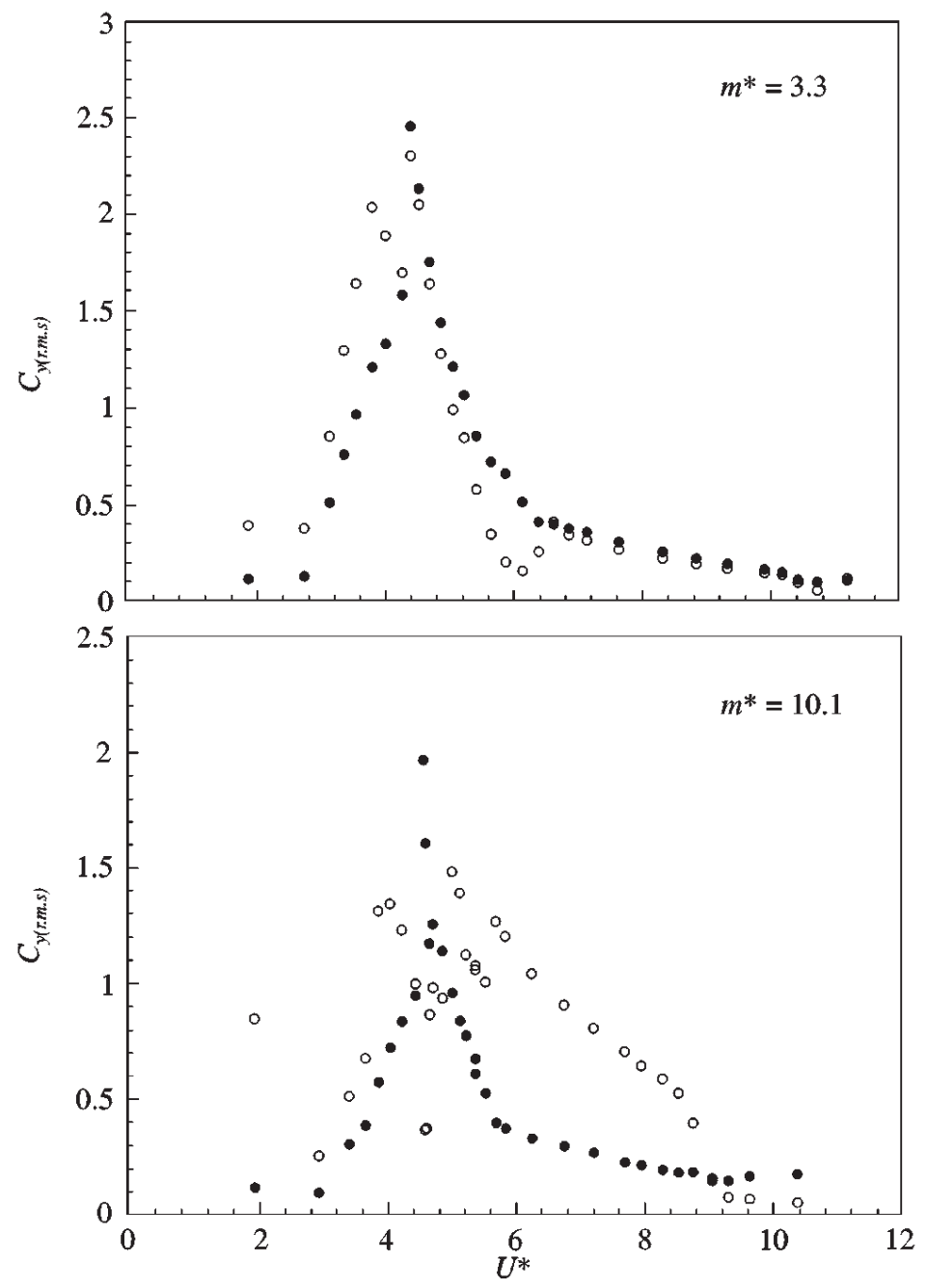

Figure 12. Estimated lift forces versus directly measured lift force as a function of normalised velocity. Good agreement is found only in the case of the lower mass ratio, $m^{*}=3 \cdot 3$. $\bigcirc$ Estimated lift force. $\bullet$ Directly measured lift force.

estimating $C_{Y}$ ensue. In conclusion, the indirect approach of estimating lift force using position response data represents a very simple method without the great difficulty in setting up a sensitive force balance, but we have shown that it is only a reasonable estimate at very low values of mass ratio.

\section{RESPONSE REGIMES}

The different branches of amplitude response are very well characterized by plotting not only the amplitude, but also the phase $\phi$ between the lift force and displacement, and the Lissajou figures or phase plane portraits of lift versus displacement, i.e. $C_{Y}(t)$ versus $Y^{*}(t)$; see Figure 13. It is clear that the jump in phase angle $\phi$ in this figure is associated with the transition from the upper branch to the lower branch. If the phase $\phi$ is defined as the lead of the lift force fluctuations over the displacement, then the upper branch $\phi$ is near $0^{\circ}$, whereas the lower branch phase is near $180^{\circ}$, rather like what is found for a linear forced system going through resonance. It is interesting that, even when the dynamics shift from the initial to upper branch, the phase angle $\phi$, as defined in equations (2) and (3), remains at just above 


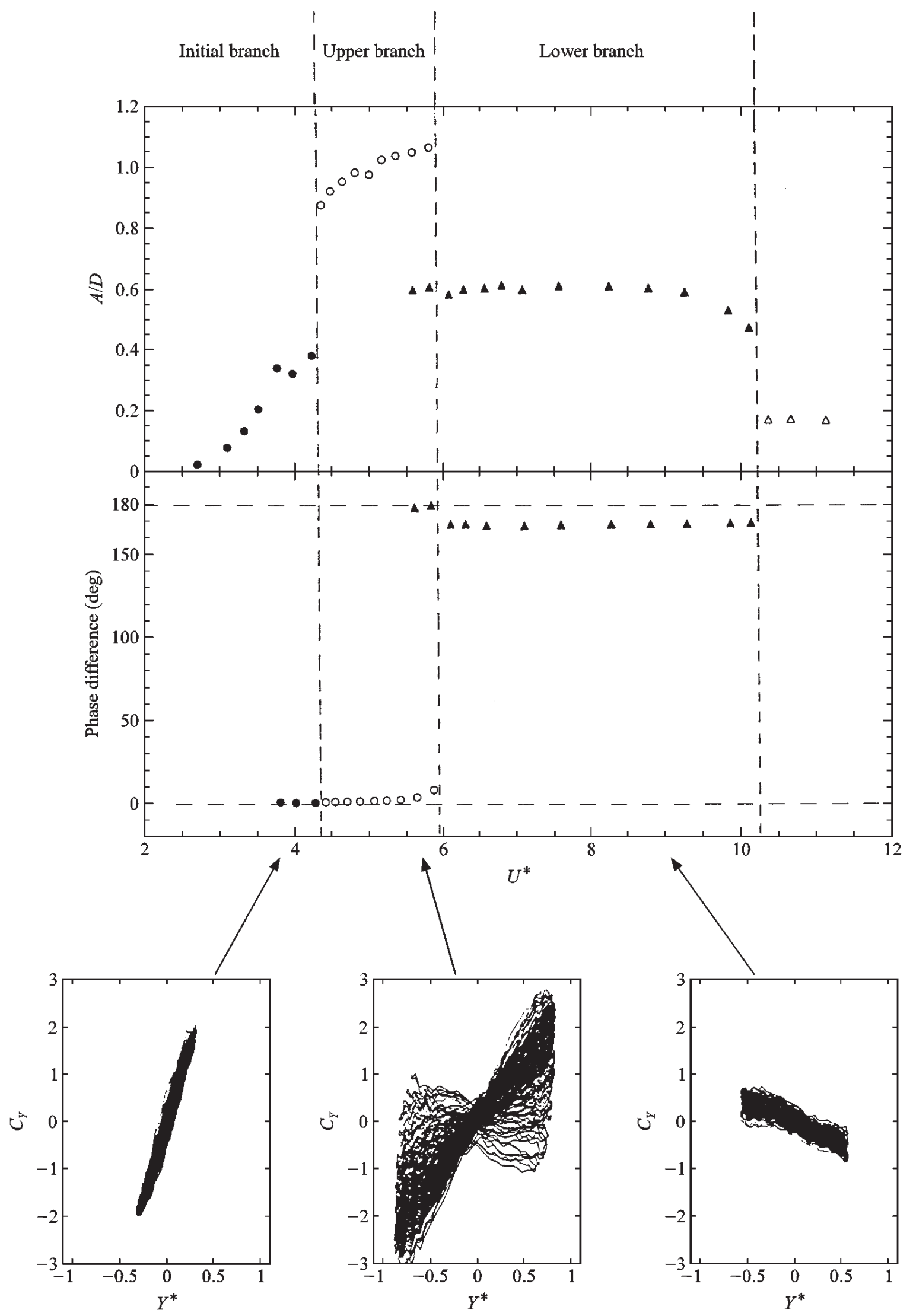

Figure 13. Characterization of the initial, upper and lower branches by their corresponding amplitudes, phase angles, and Lissajou figures (phase plots of $C_{Y}$ versus $Y^{*}$ ). The phase jump is associated with the transition from the upper to lower branch. The lower branch is remarkably steady and periodic (and exhibits the 2P mode of vortex formation). In this plot, $m^{*}=3 \cdot 3$. 
$0^{\circ}$. The Lissajou figures indicate the very periodic nature of the oscillations in the lower branch example, and the relatively less steady dynamics of the upper branch example. In the latter case, shown by the central Lissajou figure, the phase changes by around $+180^{\circ}$ as the oscillations seem to wander to the smaller amplitude of the lower branch, and then back by $-180^{\circ}$ as the oscillations switch back to the larger amplitude of the upper branch. There is an intermittent switching between the upper and lower branch amplitudes and phases, whereas we shall find a hysteretic change from the initial to the upper branch. These mode transitions are thus quite distinct.

\subsection{Initial Excitation Regime}

The Initial excitation regime is actually marked by two sub-regimes, whose existence was noted in brief by Khalak and Williamson (1997b), but not discussed there. The amplitude response, and also phase $\phi(t)$, found as a function of time by using the Hilbert transform (see Appendix A), are shown in Figure 14. The first sub-regime is characterized as "quasi-periodic" (QP in the figures), and it shows an almost periodic change in phase angle $\phi(t)$, as a function of time, as the phase "slips" periodically through $360^{\circ}$. The quasi-periodic nature of this regime is also evident in the amplitude time traces $A(t)$, again found using the Hilbert transform, as well as in the twin-peaked displacement spectra, which are included in Figure 15, for cases $U^{*}=3.46$ and 4.31 .

On the other hand, as velocity increases to $U^{*}=4 \cdot 4$, the phase angle $\phi(t)$ remains close to $0^{\circ}$, without the "slipping" through $360^{\circ}$ that was associated with the quasi-periodic regime. This sub-regime is then denoted as "periodic" ( $\mathrm{P}$ in the figures). It is evident that both of these definitions (QP and $\mathrm{P}$ ) are approximate labels, since the oscillations are not purely quasi-periodic or periodic. It can be seen in the time traces of amplitude $A(t)$, in Figure 15 that even at $U^{*}=4 \cdot 42$ and $4 \cdot 58$, some modulation of the amplitude remains, but the phase does not exhibit the "slipping" described earlier. The spectra become almost periodic as $U^{*}$ exceeds $4 \cdot 4$. As the normalized velocity, $U^{*}$, is increased still further, we enter the upper branch of response, which involves a hysteretic mode transition, as described below.

\subsection{Mode Transition: Initial $\Leftrightarrow$ Upper}

It is of particular interest to investigate the manner in which the vibrating structure can transition between one mode of response and another, and we focus here on the transition from initial excitation branch to the upper branch. In Figure 16(a), we find a distinct hysteresis between initial branch and the upper branch, as evident from response amplitude data. As $U^{*}$ velocity is increased, the jump up to the high amplitude occurs around $U^{*}=4 \cdot 70$, whereas for decreasing velocity, the drop down to the initial excitation regime occurs at close to $U^{*}=4 \cdot 50$.

Interestingly, there is also a jump in the oscillation frequency, $f^{*}$, between the initial and upper branches, as shown in Figure 17 for $m^{*}=10 \cdot 1$. As the velocity $U^{*}$ is increased, the frequency changes as follows. In the quasi-periodic initial branch, the spectra of displacement show two distinct peaks, one corresponding roughly to the vortex shedding frequency of the nonoscillating body $\left(f_{\mathrm{O}}\right)$, and one corresponding to the natural frequency $\left(f_{N}\right)$. As one enters the periodic initial branch, then only a single valued frequency is found, which is below both $f_{\mathrm{O}}$ and $f_{N}$. Further increase in $U^{*}$ to around $4 \cdot 6$, brings a jump up to an oscillation frequency at around the point where $f_{\mathrm{O}}=f_{N}$, corresponding with the start of the upper branch. At this point, the oscillation frequency $(f)$ is given by $f \approx f_{\mathrm{O}} \approx f_{N}$, and thus $f^{*} \approx 1 \cdot 0$. As the velocity $U^{*}$ is increased still further, then like the periodic initial branch, the 

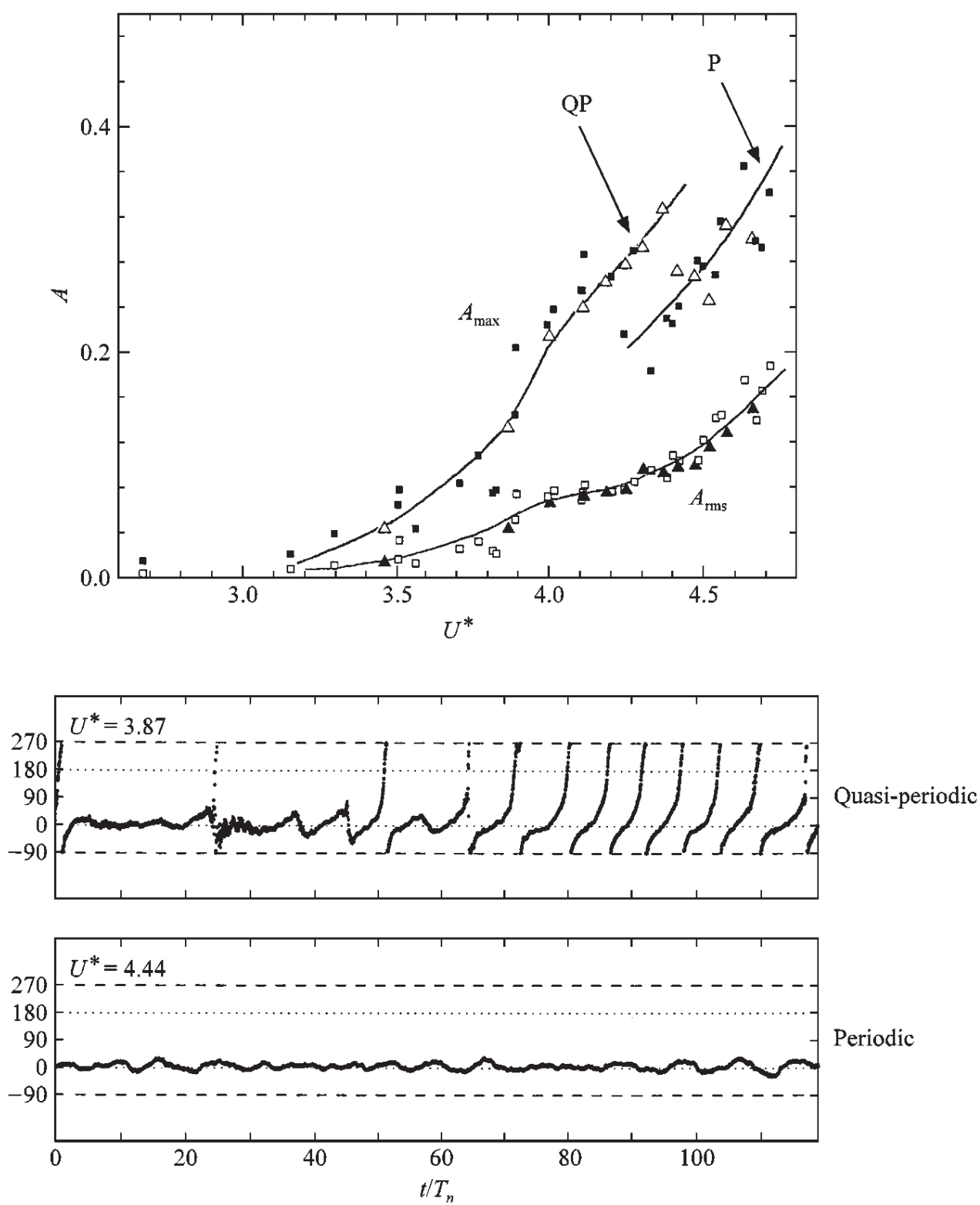

Figure 14. Initial branch. The amplitude response shows two sub-regimes of the initial branch. The quasiperiodic regime (QP) exhibits "slipping" of the phase angle $\phi(t)$ periodically through $360^{\circ}$, as a function of time, while the periodic regime shows an approximately constant phase angle $\phi$ of close to $0^{\circ}$. In the plots, the mass ratio, $m^{*}=10 \cdot 1$, and in the amplitude plot, $\zeta$ is of the order of $0 \cdot 001-0 \cdot 002$.

oscillation frequency follows neither $f_{\mathrm{O}}$ nor $f_{N}$, but in this case is somewhere between these frequencies.

These jumps in oscillation frequency become even more clear, as one reduces the mass ratio to $m^{*}=3 \cdot 3$, in Figure 18 . Here we show, not only the jump in frequency $f^{*}$ for the initial-upper branch, but also a jump in $f^{*}$ for the upper-lower branch transition. This latter transition in Figure 18 is made possible only by utilizing carefully the Hilbert transform, to properly identify the frequencies corresponding to each branch in cases when the two modes co-exist, and are intermittently switching, for the same value of velocity $U^{*}$. Further understanding of these frequency jumps is included in Govardhan \& Williamson (1999). 

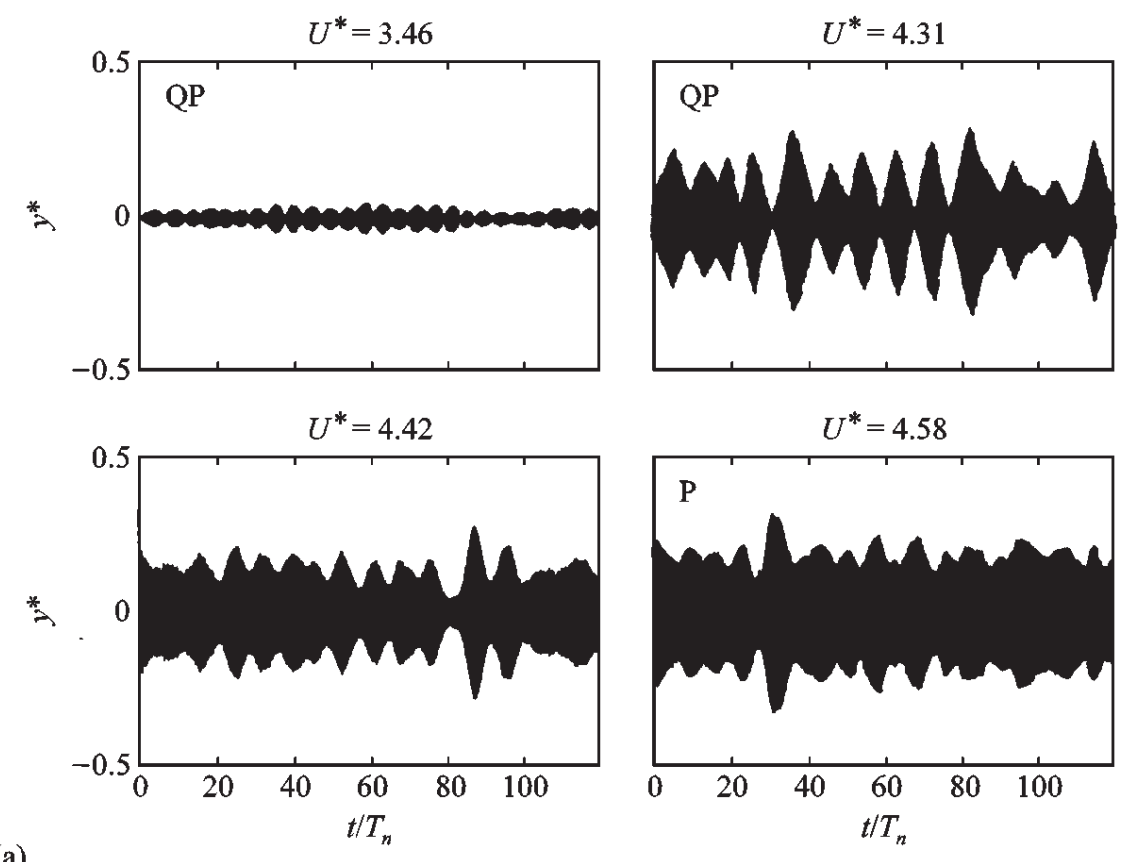

(a)
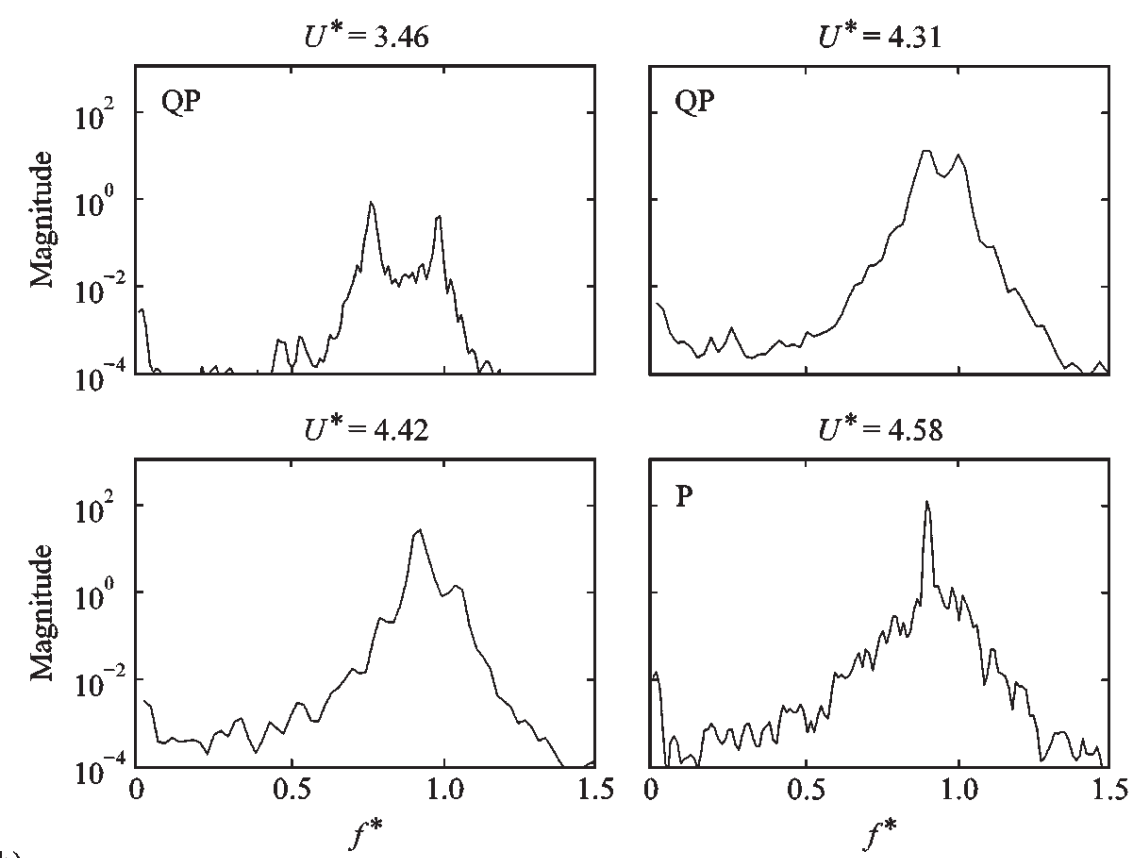

Figure 15. Initial branch amplitude $A(t)$ from the Hilbert transform, and displacement spectra, for various velocities $U^{*}$, within the initial regime of response. Quasi-periodic is denoted as QP, and periodic as $\mathrm{P}$. In this plot, $m^{*}=10.1, \zeta=0 \cdot 0013$.

\subsection{Mode Transition: Upper $\Leftrightarrow$ Lower}

The second transition, from upper to lower branches is, as already evident from the Lissajou figures in Figure 13, rather different from the first. Although there are distinctly different conditions pertaining to the upper branch versus the lower branch (different amplitudes, phase between lift and displacement, and frequency, among other parameters), the system switches between these branches intermittently, and seems only weakly "locked" into one or 

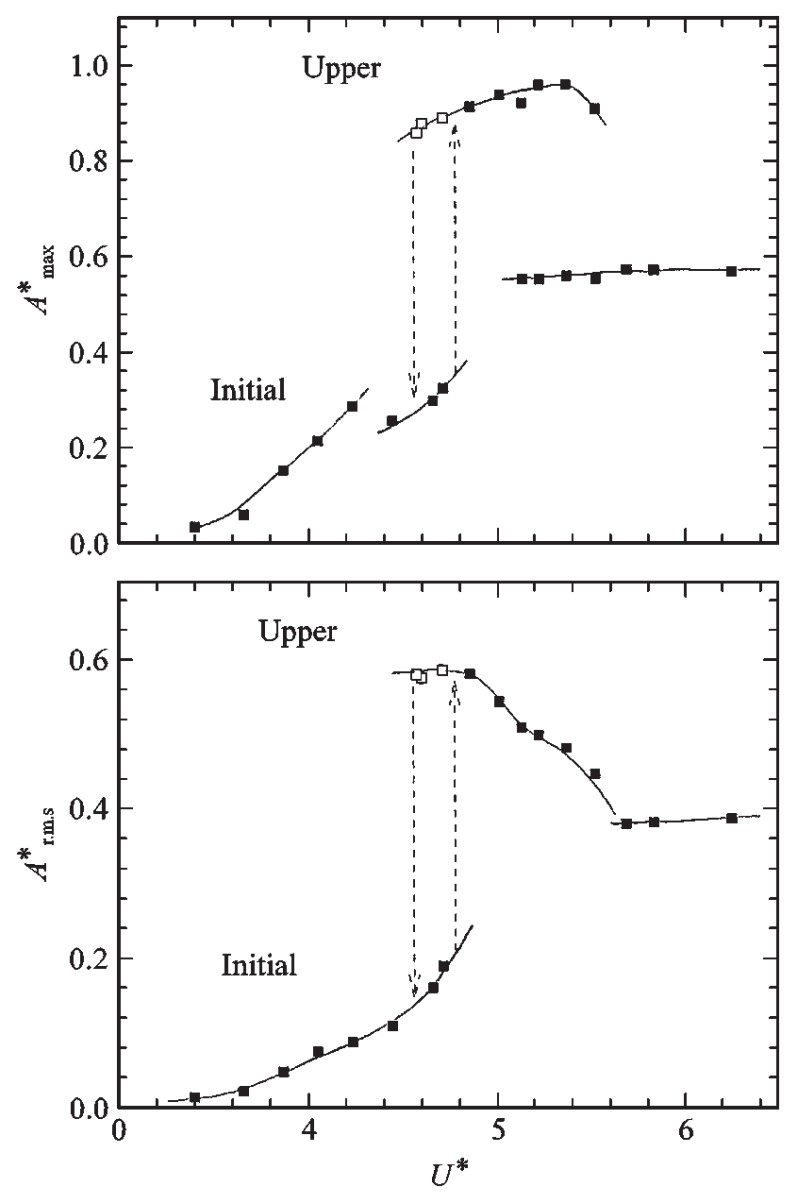

Figure 16. Mode transition: Initial $\Leftrightarrow$ upper branches, showing the jump phenomenon and hysteresis in amplitude response. In this plot, $m^{*}=10.1, \zeta=0 \cdot 0013$. Open symbols refer to the case of reducing velocity $U^{*}$.

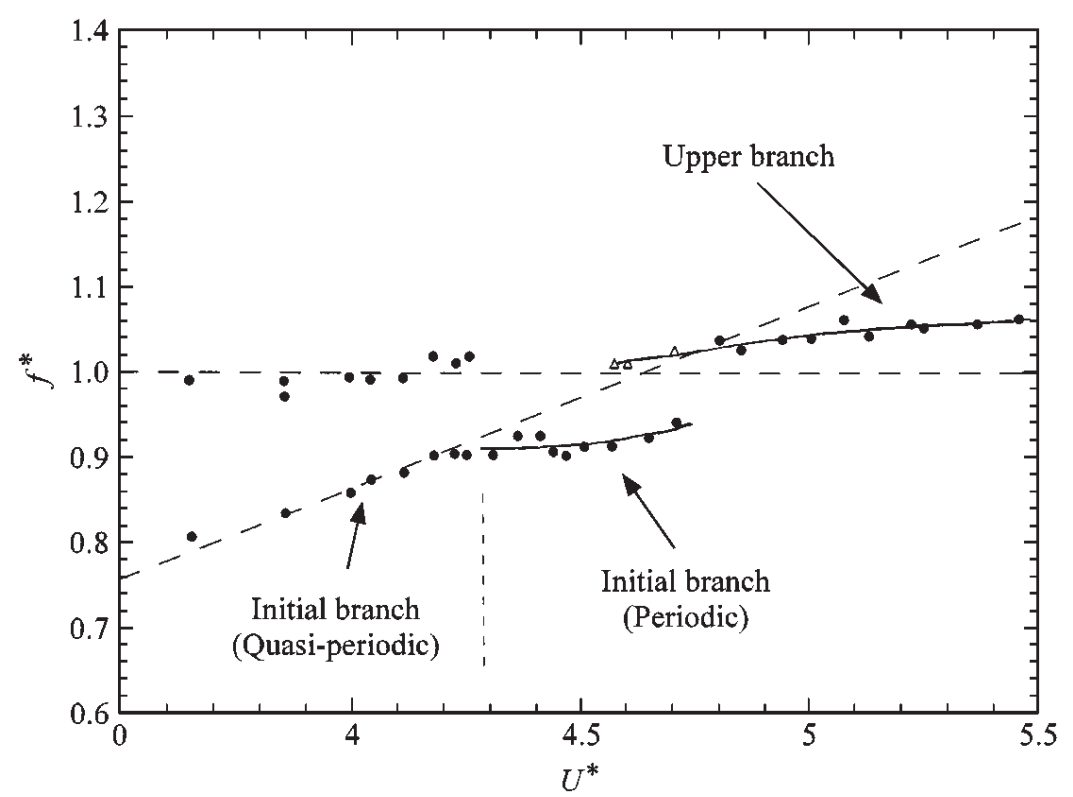

Figure 17. Mode transition: initial $\Leftrightarrow$ upper branches, showing the jump phenomenon and hysteresis in frequency response. In this plot, $m^{*}=10 \cdot 1 ; \zeta=0 \cdot 0013$. Open symbols refer to the case of reducing velocity $U^{*}$. 


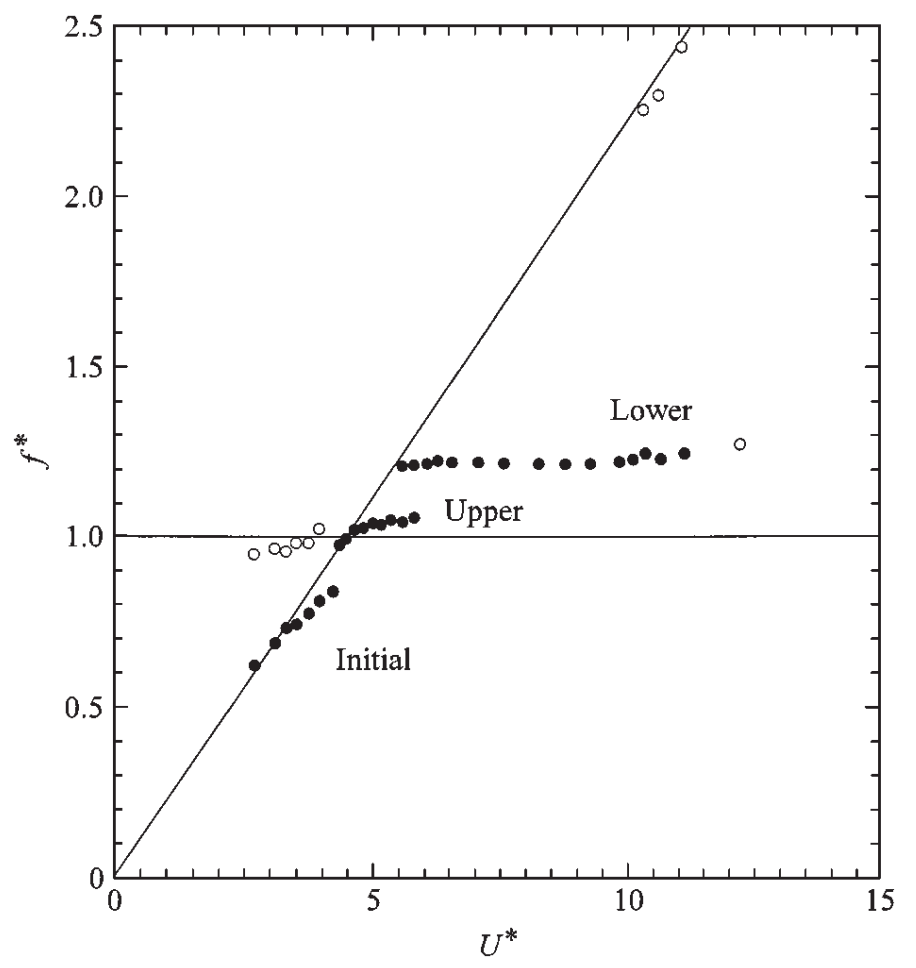

Figure 18. Both mode transitions are associated with jumps in frequency $f^{*}$, as shown in this figure. The horizontal line represents the natural frequency, when $f^{*}=1.0$ or $f=f_{N}$. The diagonal line represents the vortex-shedding frequency for the static cylinder, when $f=f_{0}$. Each solid symbol refers to the dominant spectral peak in the spectrum. In this plot, $m^{*}=3 \cdot 3, \zeta=0 \cdot 0026$.

the other mode over the range $U^{*}=5 \cdot 0-5 \cdot 6$. The intermittent switching is again demonstrated by applying the Hilbert transform to lift force-displacement data (see Appendix A). The main results relevant here are shown in Figures 19 and 20. What is interesting is that the phase, which is directly indicative of the predominant mode of oscillation (upper or lower branch type of oscillation), seems to remain for a number of periods (around 5-20 cycles) hovering around one mode, and then to (sometimes abruptly) switch to the other mode, and this scenario is clearly apparent in the central phase plot of Figure 19, when $U^{*}=5 \cdot 23$. As velocity $U^{*}$ is increased, the amount of time spent on the Upper branch mode is decreasing, while the time spent on the Lower branch mode is increasing, until at sufficiently high $U^{*}$ (see the figure for $U^{*}=5 \cdot 84$ ), the mode is stably the lower branch type all of the time. The amplitude $A(t)$ and $f^{*}(t)$ time traces in Figure 20 also show the intermittent switching, with the upper branch dominating the time sequence for $U^{*}=4 \cdot 87$, and the lower branch dominating for $U^{*}=5 \cdot 53$.

One should not be confused with the fact that the upper branch has the lower frequency $f^{*}$, and the lower branch has the upper frequency. This is also evident with the spectra in Figure 21, illustrating the dominance of the lower frequency in (a) for the upper branch, and the slightly broader spectrum associated with the switching phenomenon. When one is fully in the lower branch regime, then the spectrum is remarkably periodic; indeed the lower branch exhibits the most periodic mode of all the modes in the synchronization regime. This is an interesting final observation, particularly in light of the debate in recent conferences as to whether this mode is simply a transient phenomenon. A confirmation of the repeatability and periodicity of the steady-state lower-branch 2P mode is specifically given in Govardhan and Williamson (1999). 

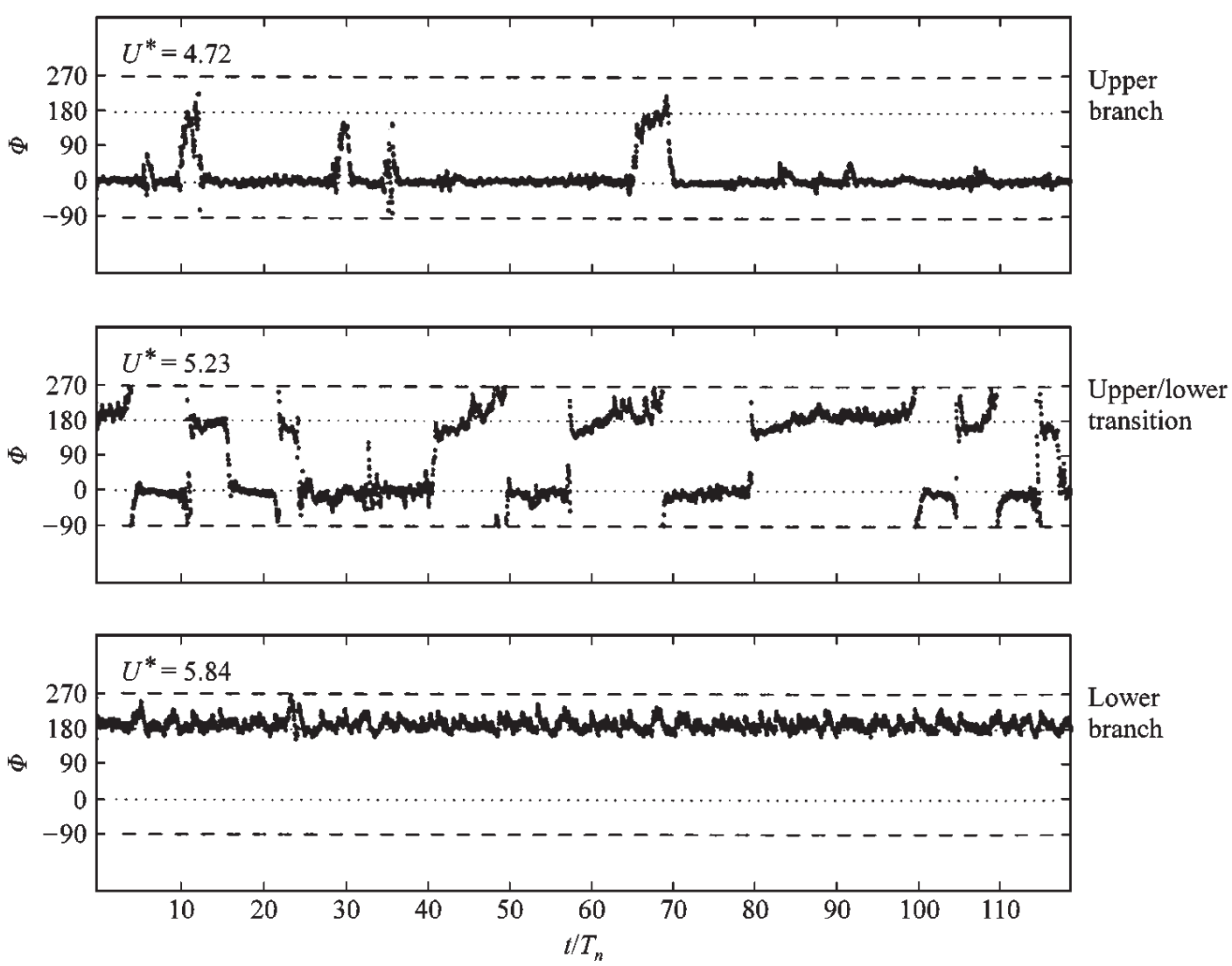

Phase, $\Phi(t)$

Figure 19. Mode transition: upper $\Leftrightarrow$ lower branches. For this second transition, we find an intermittent switching between the upper branch mode and the lower branch mode, as evidenced by the intermittent switching of the phase $\phi(t)$. This is one of the first measurements of instantaneous phase for this problem, and is made possible utilising the Hilbert transform. In this plot, $m^{*}=10 \cdot 1, \zeta=0 \cdot 0013$.

\section{CONCLUSIONS}

These experiments show that there exist two distinct types of response for the transverse oscillations of an elastically mounted rigid cylinder. At low mass-damping (low $m^{*} \zeta$ ), as one increases normalized velocity $U^{*}\left(U / f_{N} D\right)$, we find three amplitude response branches, denoted the initial branch, the upper branch, and the lower branch. At high $m^{*} \zeta$, corresponding to the classical experiments of Feng (1968), the upper branch is absent, and only two response branches exist. The peak amplitude of these vibrating systems is principally dependent on the mass-damping $m^{*} \zeta$, whereas the regime of synchronization (measured by the range of $U^{*}$ ), for a given value of $m^{*} \zeta$, is dependent primarily on the mass ratio, $m^{*}$, only.

If one employs a normalized velocity $U^{*} / f^{*}$, which is is equivalent to the "true" reduced velocity defined in recent studies (and based on the actual oscillating frequency), we find an excellent collapse of data for a set of response amplitude plots, over a wide range of mass ratios $m^{*}$. Such a collapse cannot be predicted a priori, and is only possible when $m^{*} \zeta$ is kept constant. This appears to be the first time such a collapse of data sets has been made from free vibration experiments. The response amplitude plots, in particular the response branches, match very well the Williamson-Roshko (1988) map of vortex wake patterns from forced vibration studies.

Several further questions were addressed in the introductory review of Section 1, some of which are as follows. 

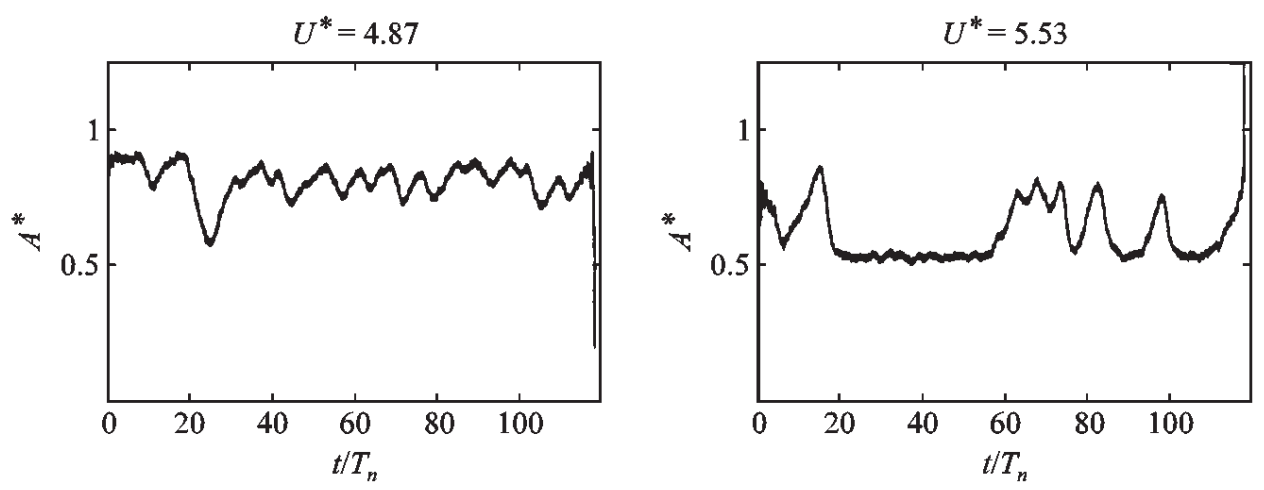

Amplitude, $A^{*}(t)$

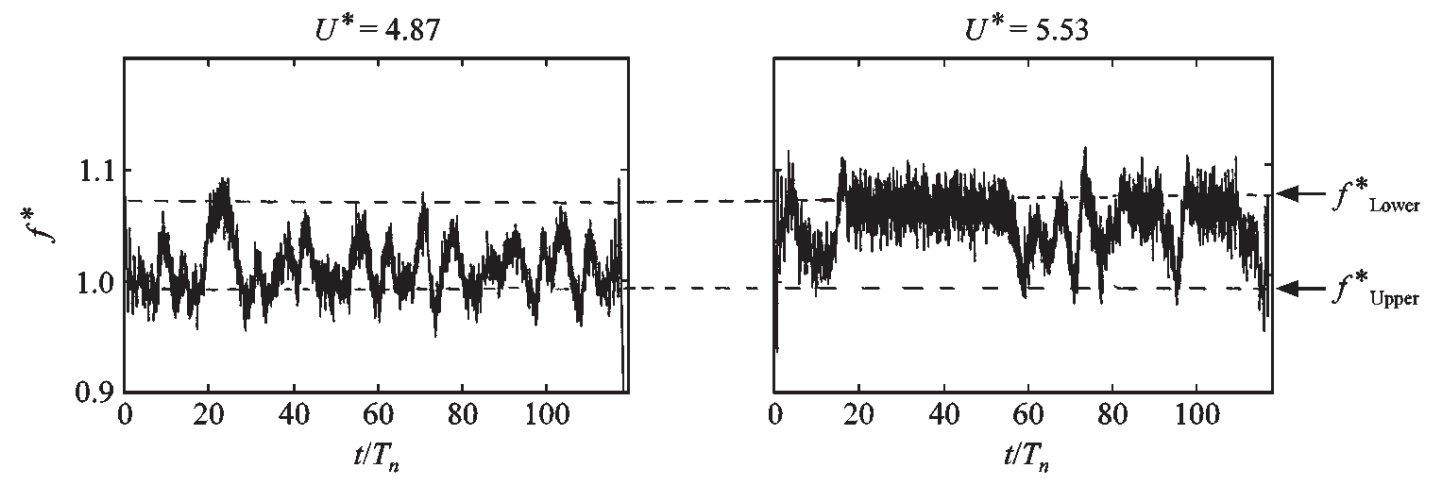

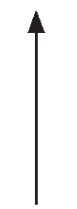

Mostly upper branch
Frequency, $f^{*}(t)$

\section{Mostly lower branch}

Figure 20. Mode transition: upper $\Leftrightarrow$ lower branches. The upper two figures show amplitude $A(t)$, and the lower two figures show $f^{*}(t)$, and they all illustrate the intermittent switching of modes at this transition. At lower $U^{*}$, more time is spent with the upper branch, while at higher $U^{*}$, more of the time is spent on the lower branch. Again, these plots are made using the Hilbert transform. In this plot, $\mathrm{m}^{*}=10 \cdot 1 ; \zeta=0 \cdot 0013$.
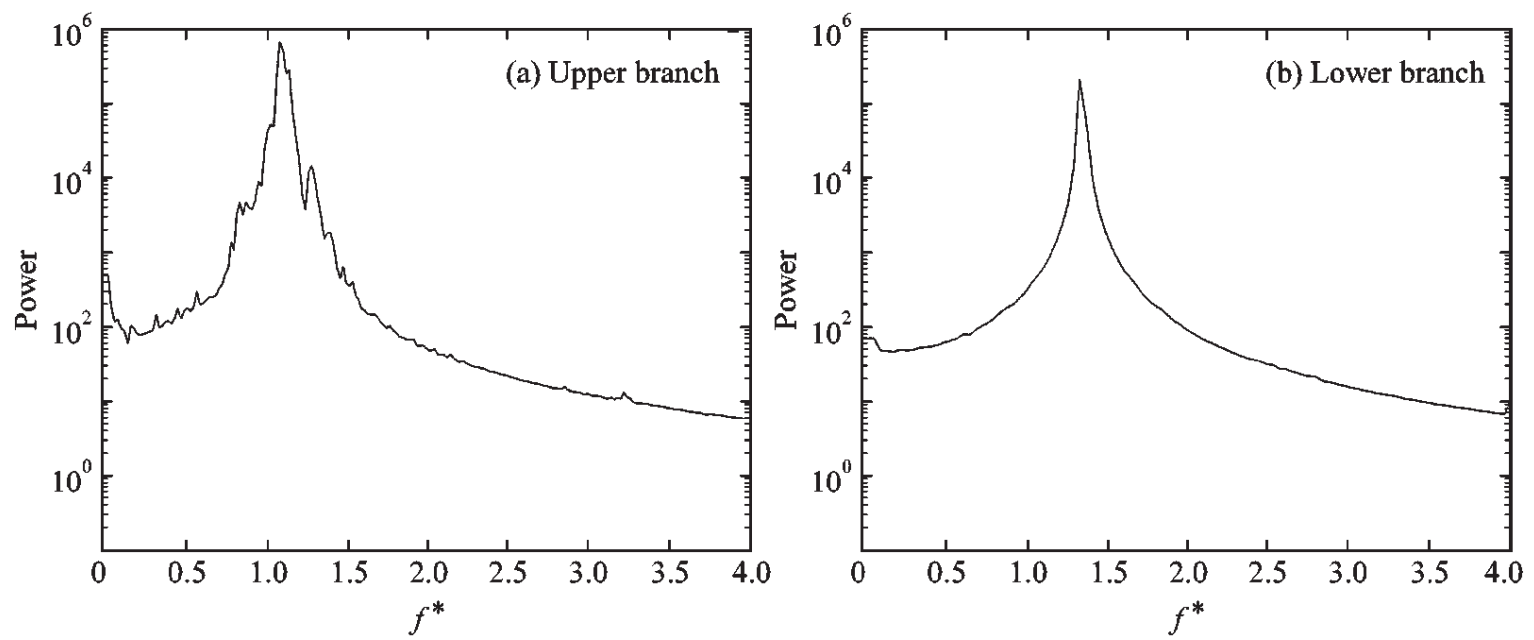

Figure 21. Spectra of displacement fluctuations, showing (a) the almost periodic upper branch, and (b) the remarkably periodic lower branch (associated with the $2 \mathrm{P}$ mode of vortex formation). 
How do the mode transitions occur? The transitions between the modes of response are discontinuous. At low $m^{*} \zeta$, the mode change between initial and upper response branches involves a hysteresis. This contrasts with the intermittent switching of modes found for the transition between upper-lower branches. The temporal fluctuations of phase angle $\phi$, response amplitude $A^{*}$, and frequency $f^{*}$, have been found utilizing the Hilbert transform, which allows us to clearly illustrate these transitions, in a manner not otherwise possible using averaged quantities. Both of the mode transitions are associated with jumps in amplitude and frequency, but the $180^{\circ}$ jump in phase angle $\phi$ is found only when the flow jumps between the upper-lower branches of response.

What are the wake vortex dynamics for each mode? Flow visualization of the modes indicates that the initial branch is associated with the $2 \mathrm{~S}$ mode of vortex formation, while the lower branch corresponds with the $2 \mathrm{P}$ mode [confirming the visualizations of Brika $\&$ Laneville (1993)]. There has been some debate recently as to whether the $2 \mathrm{P}$ mode is a transient phenomenon or is a steady-state wake mode. Interestingly, the present work suggests that the $2 \mathrm{P}$ mode is the most periodic of any of the wake patterns in the synchronization regime. However, the present flow visualization and response/force data are not sufficient to prove repeatability, or to clarify the vortex mode for the upper branch. These points are specifically addressed in Govardhan \& Williamson (1999), where the use of the DPIV technique yields clearly the vorticity dynamics.

Under what conditions does the classically employed "mass-damping parameter" collapse peak amplitude data? One of the problems in answering this question to-date has been in comparing data coming from quite dissimilar experiments from the last 20 years. The large scatter in such data has been masked to some extent by the standard use of "log-log" plots for presenting peak ampitude data. It was our intention to conduct a set of experiments, over a range of $m^{*}$ and $\zeta$, but under the same experimental conditions, to determine the validity of using the combined parameter $\left(m^{*}+C_{A}\right) \zeta$. The excellent collapse of peakamplitude data, over a wide range of mass ratios $\left(m^{*}=1-20\right)$, when plotted against $\left(m^{*}+C_{A}\right) \zeta$ in the "Griffin" plot, demonstrates that the use of a combined parameter is valid down to at least $\left(m^{*}+C_{A}\right) \zeta \sim 0.006$. This is two orders of magnitude below the "limit" that had previously been stipulated in the literature $\left[S_{G}>1\right.$, or equivalently $\left.\left(m^{*}+C_{A}\right) \zeta>0 \cdot 4\right]$. One expects, nevertheless, that there will exist conditions under which the mass $m^{*}$ will independently influence the peak response amplitude, even when $m^{*} \zeta$ is kept constant.

What is the maximum attainable amplitude for a cylinder undergoing vortex-induced vibrations, for conditions of extremely low mass and damping? A firm numerical answer to this question is not yet available from experiment. In the present study, the peak amplitude is found to increase steadily as we reduce mass-damping, reaching a value $A^{*}=1 \cdot 20$. This exceeds all peak values from previous experiments of this type, and we find no signs yet of amplitude saturation. Interestingly, in the Griffin plot, our amplitude data are found to cross over, and do not appear to follow even qualitatively, recent empirical curve fits for accumulated data from previous vortex-induced vibration experiments. One can say, at this point, that the maximum attainable amplitude $\left(A^{*}\right)$ will be larger than 1.20 for a massdamping $\left(m^{*}+C_{A}\right) \zeta$ of less than $0 \cdot 006$. A precise evaluation of this maximum amplitude is the subject of ongoing research. One would imagine that computer simulation, where it is possible to set damping precisely to zero, would yield the largest possible amplitude. However, the low Re in recent simulations yield wake dynamics and response markedly different from the high-Re experiments, and maximum amplitudes are only around $A^{*}=0 \cdot 6$. 
Further work is being conducted to probe the limits of the absolute largest amplitude that is attainable for such a system, at extremely low values of mass and damping, and to complete the set of peak-amplitude data defining the Griffin plot. It is expected that some form of limit to the mass ratio will be found, beyond which the combined mass-damping $m^{*} \zeta$ will not, on its own, be able to define the peak amplitude. Much further work remains to be done concerning a clear understanding of the fluid physics of the vortex wake modes, and their effect on the force and dynamics of the structure, amongst other studies.

\section{ACKNOWLEDGEMENTS}

Immense thanks are due to Raghuraman Govardhan for superb help and enthusiasm with this work, and for his rare joie de vivre. The support from the Ocean Engineering Division of the O.N.R., monitored by Dr Tom Swean, is gratefully acknowledged (O.N.R. Contract Nos. N00014-94-1-1197 and N00014-95-1-0332).

\section{REFERENCES}

Anagnostopoulos, P. \& Bearman, P. W. 1992 Response characteristics of a vortex-excited cylinder at low Reynolds numbers. Journal of Fluids and Structures 6, 39-50.

Anand, N. M. \& Torum, A. 1985 Free span vibration of submerged pipelines in steady flow and waves. In Proceedings of International Symposium on Separated Flow Atround Marine Structures, pp. 155-199, Trondheim, Norway.

Angrilli, F. Disilvio, G. \& Zanardo, A. 1974 Hydroelasticity study of a circular cylinder in a water stream. In Flow Induced Structural Vibrations (ed. E. Naudascher), pp. 504-512. Berlin: Springer-Verlag.

Atsavapranee, P., Benaroya, H. \& Wei, T. 1998 Vortex dynamics in the near wake of an oscillating cylinder. In Paper Proceedings Conference on Bluff Body Wakes and Vortex-Induced Vibrations, Washington, DC, 21-23 June 1998 (eds P. W. Bearman \& C. H. K. Williamson), Paper Number 5; also Paper FEDSM98-5170 in CD-ROM from ASME.

Balasubramanian, S. \& Sкop, R.A. 1996 A nonlinear oscillator model for vortex shedding from cylinders and cones in uniform and shear flows. Journal of Fluids and Structures 10, 197-214.

Bearman, P. W. 1984 Vortex shedding from oscillating bluff bodies. Annual Review of Fluid Mechanics 16, 195-222.

Bearman, P. W. \& Williamson, C. H. K. (eds) 1998 Paper Proceedings Conference on Bluff Body Wakes and Vortex-Induced Vibrations, Washington, DC, 21-23 June 1998. Printed at Cornell University, NY (Also, in highly segmented form on CD-ROM for the ASME Fluids Engineering Summer Meeting, FEDSM98.)

Blackburn, H. \& Henderson, R. 1995 Wake dynamics in flow past an oscillating cylinder. In 9th Conference on Numerical Methods in Laminar and Turbulent Flow, Atlanta, GA, U.S.A., July 1995.

Blackburn, H. \& Karniadakis, G. E. 1993 Two and Three dimensional simulations of vortex-induced vibration of a circular cylinder. In 3rd International Offshore and Polar Engineering Conference, Vol. 3, pp. 715-720.

Blevins, R. D. 1990 Flow-Induced Vibrations. New York: Van Nostrand Reinhold.

Bracewell, R. 1986. The Fourier Transform and its applications. New York: McGraw-Hill.

Brika, D. \& LANeville, A. 1993 Vortex-induced vibrations of a long flexible circular cylinder. Journal of Fluid Mechanics 250, 481-508.

Cui, E. J. \& Dowell, E. H. 1981 An approximate method for calculating the vortex-induced oscillation of bluff bodies in air and water. In Proceedings of Structures and Materials Conference, ASME Winter Annual Meeting, Washington, DC, 15-20 November pp. 155-173.

Dean, R. B., Milligan, R. W. \& Wootton, L.R. 1977 Study of flow-induced vibration. Atkins Research and Development Report, London, U.K.

Evangelinos, C. \& Karniadakis, G. E. 1998 Dynamics and flow structures in a turbulent wake of rigid and flexible cylinders subject to vortex-induced vibration. Journal of Fluid Mechanics (submitted). 
FENG, C. C. 1968 The measurement of vortex-induced effects in flow past a stationary and oscillating circular and D-section cylinders. Master's Thesis, University of British Columbia, Vancouver, B.C., Canada.

Fujarra, A. L. C., Meneghini, J. R., Pesce, C. P. \& Parra, P. H. C. C. 1998 An investigation of vortex-induced vibration of a circular cylinder in water. In Paper Proceedings Conference on Bluff Body Wakes and Vortex-Induced Vibrations, Washington, DC, 21-23 June 1998. (eds P. W. Bearman \& C. H. K. Williamson), Paper Number 13; also Paper FEDSM98-5195 in CD-ROM from ASME.

Gharib, M. R., Leonard, A., Gharib, M. \& Roshko, A. 1998. The absence of lock-in and the role of mass ratio. In Paper Proceedings Conference on Bluff Body Wakes and Vortex-Induced Vibrations, Washington, DC, 21-23 June 1998. (eds P. W. Bearman \& Williamson C. H. K.), Paper Number 24; also Paper FEDSM98-5312 in CD-ROM from ASME.

Gopalkrishnan, R. 1993 Vortex-induced forces on oscillating bluff cylinders. Ph.D. Thesis, MIT, Cambridge, MA, U.S.A.

Govardhan, R. \& Williamson, C. H. K. 1997 Vortex induced motions of a tethered sphere. Journal of Wind Engineering and Industrial Aerodynamics 69-71, 375-385.

Govardhan, R. \& Williamson, C. H. K. 1998 2P or not 2P. American Physical Society-51st Annual Meeting of the DFD. Conference Summary, p. 11. Philadelphia, 22-24 November

Govardhan, R. \& Williamson, C. H. K. 1999 Modes of vortex formation and frequency response for a freely-vibrating cylinder. Journal of Fluid Mechanics (submitted).

Griffin, O. M. 1985 Vortex shedding from bluff bodies in a shear flow. ASME Journal of Fluids Engineering 107, 298-306.

Griffin, O. M. 1980 Vortex-excited cross-flow vibrations of a single cylindrical tube. ASME Journal of Pressure Vessel Technology 102, 158-166.

Griffin, O. M., Skop, R. A. \& Ramberg, S. E. 1975 The resonant vortex-excited vibrations of structures and cable systems. In 7th Offshore Technology Conference. OTC Paper 2319. Houston, Texas, U.S.A.

Griffin, O. M. \& RamberG, S. E. 1974 The vortex street wakes of vibrating cylinders. Journal of Fluid Mechanics 66, 553-576.

Griffin, O. M. \& Ramberg, S. E. 1982 Some recent studies of vortex shedding with application to marine tubulars and risers. ASME Journal of Energy Resources Technology 104, 2-13.

Gu, W, Chyu, C. \& Rockwell, D. 1994 Timing of vortex formation from an oscillating cylinder. Physics of Fluids 6, 3677-3682.

Hover, F. S., Miller, S. N. \& Triantafyllou, M. S. 1997 Vortex-induced vibration of marine cables: experiments using force feedback. Journal of Fluids and Structures 11, 307-326.

Hover, F. S., Techet, A. H. \& Triantafyllou, M. S. 1998 Forces on oscillating uniform and tapered cylinders in crossflow. Journal of Fluid Mechanics 363, 97-114.

Hover, F. S. \& Triantafyllou, M. S. 1999 Spanwise vortex structures behind a vibrating cylinder in crossflow. Submitted to Journal of Fluids and Structures.

Khalak, A. \& Williamson, C. H. K. 1996 Dynamics of a hydroelastic cylinder with very low mass and damping. Journal of Fluids and Structures 10, 455-472.

Khalak, A. \& Williamson, C. H. K. 1997a Investigation of the relative effects of mass and damping in vortex-induced vibration of a circular cylinder. Journal of Wind Engineering and Industrial Aerodynamics 69-71, 341-350.

Khalak, A. \& Williamson, C. H. K. 1997b Fluid forces and dynamics of a hydroelastic structure with very low mass and damping. Journal of Fluids and Structures 11, 973-982.

KING, R. 1974 Vortex-excited structural oscillations of a circular cylinder in flowing water. Ph.D. Thesis, Loughborough University of Technology, Loughborough, England.

Lu, X-Y. \& Dalton, C. 1996 Calculation of the timing of vortex formation from an oscillating cylinder. Journal of Fluids and Structures 10, 527-541.

Meier-Windhorst, A. 1939 Flatterschwingungen von Zylindern im gleichmassigen Flussigkeitsstrom. Mitteilungen des Hydraulischen Instituts der Technischen Hochschule, München 9, 3-39.

Meneghini, J. R. \& Bearman, P. W. 1995. Numerical simulation of high amplitude oscillatory flow about a circular cylinder. Journal of Fluids and Structures 9, 435-455.

Mercier, J. A. 1973 Large amplitude oscillations of a circular cylinder in a low speed stream. Ph.D. Thesis, Stevens Institute of Technology, NJ, USA.

Moe, G. \& Wu, Z-J. 1990 The lift force on a cylinder vibrating in a current. ASME Journal of Offshore Mechanics and Arctic Engineering 112, 297-303. 
Moe, G. \& OverviK, T. 1982 Current-induced motions of multiple risers. In Proceedings of BOSS-82, Behaviour of Offshore Structures, Vol. 1, pp. 618-639. Cambridge, MA, U.S.A.

Naudascher, E. \& Rockwell, D. 1993 Flow-Induced Vibrations: An Engineering Guide. Rotterdam: Balkema.

Newman, D. \& Karniadakis, G. E. 1996 Simulations of flow over a flexible cable: Comparison of forced and flow-induced vibration. Journal of Fluids and Structures 10, 439-453.

Ongoren, A. \& Rockwell, D. 1988 Flow structure from an oscillating cylinder. Part 2. Mode competition in the near wake. Journal of Fluid Mechanics 191, 225-245.

Parkinson, G. 1989 Phenomena and modelling of flow-induced vibrations of bluff bodies. Progress in Aerospace Sciences 26, 169-224.

Pesce, C. P. \& Fujarra, A. L. C. 1999 Vortex-induced vibrations and jump phenomenon: experiments with a clamped flexible cylinder in water. International Journal of Offshore and Polar Engineering (to appear).

Ramberg, S. E. \& Griffin, O. M. 1981 Hydroelastic response of marine cables and risers. In Hydrodynamics in Ocean Engineering, pp. 1223-1245. Norwegian Institute of Technology, Trondheim, Norway.

Saltara, F., Meneghini, J. R., Siqueira, C. R. \& Bearman, P. W. 1998 The simulation of vortex shedding from an oscillating circular cylinder with turbulence modelling. In Bearman and Williamson (1998), Paper Number 25; also Paper FEDSM98-5189 in CD-ROM from ASME.

Sarpkaya, T. 1978 Fluid forces on oscillating cylinders. ASCE Journal of Waterway, Port, Coaastal, and Ocean Division 104, 275-290.

Sarpkaya, T. 1979 Vortex-induced oscillations. Journal of Applied Mechanics 46, 241-258.

SARPKAYA, T. 1993 Offshore hydrodynamics. ASME Journal of Offshore Mechanics and Arctic Engineering 115, 2-5.

SARPKAYA, T. 1995 Hydrodynamic damping, flow-induced oscillations, and biharmonic response. ASME Journal of Offshore Mechanics and Arctic Engineering 117, 232-238.

Scruton, C. 1965 On the wind-excited oscillations of towers, stacks and masts. In Proceedings of the Symposium on Wind Effects on Buildings and Structures, Paper 16, pp. 798-836. London: Her Majesty's Stationery Office.

Sheridan, J., CARberry, J., Lin, J.-C. \& Rockwell, D. 1998. On the near wake topology of an oscillating cylinder. Journal of Fluids and Structures 12, 215-220.

Shiels, D., Leonard, A. \& Roshro, A. 1998 Flow-induced vibration of a circular cylinder at limiting structural parameters. Journal of Fluids and Structures (submitted).

Skop, R. A. 1974 On modelling vortex-excited oscillations. NRL Memorandum Report 2927.

Skop, R. A. \& Balasubramanian, S. 1997 A new twist on an old model for vortex-excited vibrations. Journal of Fluids and Structures 11, 395-412.

Sкоp, R. A. \& Griffin, O. M. 1973 An heuristic model for determining flow-induced vibrations of offshore strcutures. In 5th Offshore Technology Conference, OTC Paper 1843, Houston, Ts, U.S.A.

Stansby, P. K. 1976 The locking-on of vortex shedding due to the cross-stream vibration of circular cylinders in unform and shear flows. Journal of Fluid Mechanics 74, 641-655.

Staubl, T. 1983 Calculation of the vibration of an elastically-mounted cylinder using experimental data from forced oscillation. ASME Journal of Fluids Engineering 105, 225-229.

Sumer, B. M. \& Fredsøze, J. 1997 Hydrodynamics around Cylindrical Structures. Singapore: World Scientific.

Techet, A. H., Hover, F. S. \& Triantafyllou, M. S. 1998 Vortical patterns behind a tapered cylinder oscillating transversely to a uniform flow. Journal of Fluid Mechanics 363, 79-96.

Triantafyllou, M. S. \& Grosenbaugh. 1995 Prediction of vortex-induced vibrations in sheared flows. In Flow-Induced Vibration (ed. P.W.Bearman), pp. 73-82. Rotterdam: Balkema.

VICKERY, B. J. \& WATKINS, R. D. 1964 Flow-induced vibrations of cylindrical structures. In Proceedings of the First Australian Conference on Hydraulics and Fluid Mechanics (ed. R. Silvester). New York: Pergamon Press.

VIKESTAD. 1998 Multi-frequency response of a cylinder subjected to vortex shedding and support motions. Ph.D. Thesis Norwegian Institute of Technology, Trondheim, Norway. Data provided to the authors by K. Vandiver, Private Communication.

Williamson, C. H. K. \& Roshko, A. 1988. Vortex formation in the wake of an oscillating cylinder. Journal of Fluids and Structures 2, 355-381.

Zdravkovich, M. M. 1982 Modification of vortex shedding in the synchronization range. ASME Journal of Fluids Engineering 104, 513-517. 
ZDRAVKovich, M. M. 1990 On origins of hysteretic responses of a circular cylinder induced by vortex shedding. Zeitschrift fur Flugwissenschaften Weltraumforschung 14, 47-58.

\section{APPENDIX A: COMPUTATION OF INSTANTANEOUS AMPLITUDE AND PHASE BY MEANS OF THE HILBERT TRANSFORM}

The definitions of instantaneous amplitude and instantaneous phase of the signal are intuitive, but are not obvious to precisely define. We can generate precise definitions for these quantities using the Hilbert transform, which is related to the Fourier transform. An extensive treatment of transforms is given in Bracewell (1986).

A single complex-valued quantity can embody both instantaneous amplitude and instantaneous phase. That is, for a real-valued signal, $x(t)$, we can define $z(t)$ a complex-valued signal such that the amplitude and phase of $z(t)$ are actually the instantaneous amplitude and phase of $x(t)$. That we discuss the instantaneous phase of the signal indicates that this procedure is relevant for signals that are primarily monochromatic. For signals which do not have a dominant frequency, more general techniques such as wavelet analysis may be more applicable.

We impose the constraint on $z(t)$ that its phase should be monotonic with time. That is, if we follow $z(t)$ in the complex plane as it rotates about zero, corresponding to the oscillations in $x(t)$, then the sense of the rotation should always be counter-clockwise. Continuing the image of $z(t)$ in the complex plane, the instantaneous amplitude is simply the distance of $z(t)$ from the origin. For example, one may construct the complex-valued signal $z(t)=\mathrm{e}^{\mathrm{i} \omega t}$ from the real-valued signal $x(t)=\cos (\omega t)$.

A monotonically increasing phase demands that the frequency content of $z(t)$ be solely positive. This can be generated from the original signal, $x(t)$, as follows. Define $X(\omega) \equiv \mathscr{F}(x)$, where $\mathscr{F}$ denotes the operator for the Fourier transform. Then, define $z(t)$ such that $Z(\omega) \equiv \mathscr{F}(z)$ has the property

$$
Z(\omega)= \begin{cases}2 X(\omega), & \omega \geqslant 0 \\ 0 & \omega<0\end{cases}
$$

This can also be written as

$$
Z(\omega)=X(\omega)+\operatorname{sgn}(\omega) X(\omega),
$$

where sgn is the signum function. The quantity $z(t)$, given $x(t)$, then becomes

$$
\begin{aligned}
z(t) & \equiv \mathscr{F}^{-1}\{X(\omega)+\operatorname{sgn}(\omega) X(\omega)\} \\
& =x(t)+\mathscr{F}^{-1}\{\operatorname{sgn}(\omega) X(\omega)\} .
\end{aligned}
$$

To continue further, we need to introduce the Hilbert transform, $\mathscr{H}$, which is defined as

$$
\mathscr{H}(x) \equiv \frac{1}{\pi} \int_{-\infty}^{\infty}\left\{\frac{x(t)}{t-s}\right\} \mathrm{d} t,
$$

where $s$ is the transformed variable. Since this integral has a singularity at $t=s$, we take the Cauchy principle value (i.e. around a contour integral in the complex plane on a path which is perturbed near $t=s$ ). The Hilbert transform can also be written in terms of a convolution

$$
\mathscr{H}(x)=\frac{-1}{\pi t} * x(t) .
$$

where $*$ represents the convolution operator. We can rewrite the Hilbert transform in terms of the more familiar Fourier transform as follows:

$$
\begin{aligned}
\mathscr{H}(x) & =\mathscr{F}^{-1}\left\{\mathscr{F}\left(\frac{-1}{\pi t} * x\right)\right\} \\
& =\mathscr{F}^{-1}\left\{\mathscr{F}\left(\frac{-1}{\pi t}\right) \mathscr{F}(x)\right\} \\
& =-\mathrm{i}^{-1}\{\operatorname{sgn}(\omega) \mathscr{F}(x)\}
\end{aligned}
$$




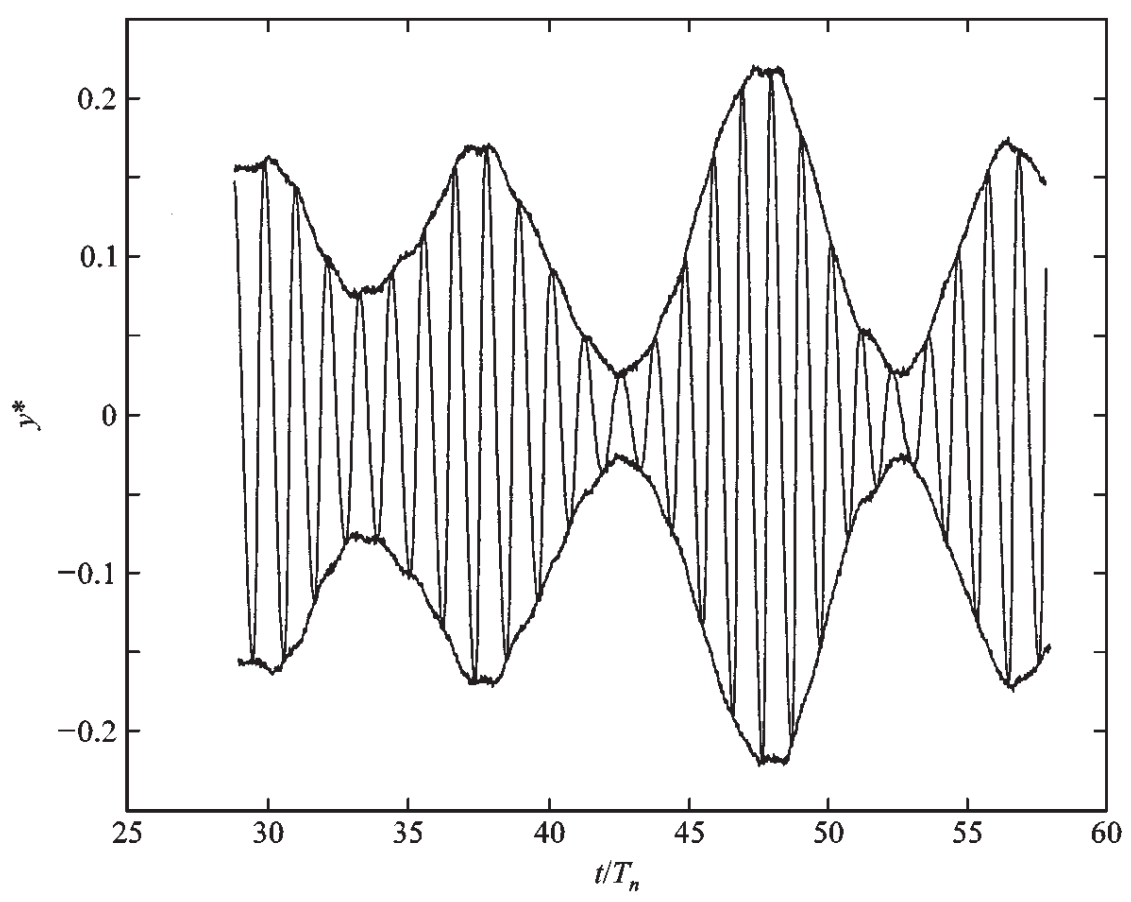

Figure A1. Instantaneous amplitude calculated by means of the Hilbert transform. This example is for the Initial excitation regime, $U^{*}=4 \cdot 23$.

where $\mathscr{F}$ is the Fourier transform and $\omega$ is the transform variable. Substituting the expression for $\mathscr{H}(x)$, equation (A6), into the expression for $z(t)$, equation (A2), we obtain

$$
z=x-\mathrm{i} \mathscr{H}(x) \text {. }
$$

Thus, we can find the instantaneous amplitude and phase of the signal $x(t)$ as the amplitude and phase of the complex-valued signal $z(t)$, defined by equation (A7). Figure A.1 gives a pictorial example of the demodulation procedure on an oscillatory time trace with time-varying amplitude typical of those measured in our experiments. 